\title{
Exactly solvable models in atomic and molecular physics
}

\author{
A. Foerster ${ }^{\mathrm{a}}$, E. Ragoucy ${ }^{\mathrm{b}, *}$ \\ a Instituto de Física da UFRGS, Av. Bento Gonçalves 9500, Porto Alegre, RS, Brazil \\ ${ }^{\mathrm{b}}$ Laboratoire de Physique Théorique LAPTH, LAPP, BP 110, F-74941 Annecy-le-Vieux cedex, France
}

Received 8 February 2007; accepted 21 March 2007

Available online 18 April 2007

\begin{abstract}
We construct integrable generalized models in a systematic way exploring different representations of the $g l(N)$ algebra. The models are then interpreted in the context of atomic and molecular physics, most of them related to different types of Bose-Einstein condensates. The spectrum of the models is given through the analytical Bethe ansatz method. We further extend these results to the case of the superalgebra $g l(M \mid N)$, providing in this way models which also include fermions.
\end{abstract}

(c) 2007 Elsevier B.V. All rights reserved.

PACS: 75.10.Jm; 71.10.Fd; 03.65.Fd

\section{Introduction}

Exactly solvable models are a fascinating issue that continue to attract considerable interest in physics and mathematics. Although the integrability of quantum systems is usually restricted to one dimension, there are many reasons that turn this study relevant for physical applications. It serves as a test for computer analysis and analytical methods for realistic systems to which, until now, only numerical calculations and perturbative methods may be applied. In addition, a nontrivial solvable model reveals the essence of the phenomena under consideration. For instance, many concepts established in critical phenomena were inspired by the exact solution of the Ising model. From the experimental point of view, there are some real spin-1 compounds (e.g. NENC, NDPK, or NBYC, etc.) [1] and strong coupling ladder compounds (such as (5IAP) ${ }_{2} \mathrm{CuBr}_{4} \cdot 2 \mathrm{H}_{2} \mathrm{O}$,

\footnotetext{
* Corresponding author.

E-mail addresses: angela@if.ufrgs.br (A. Foerster), ragoucy@lapp.in2p3.fr (E. Ragoucy).
} 
or $\mathrm{Cu}_{2}\left(\mathrm{C}_{5} \mathrm{H}_{12} \mathrm{~N}_{2}\right)_{2} \mathrm{Cl}_{4}$, etc.) [2] that can be well described by integrable models. The necessity of using exactly solvable models has been also demonstrated through experimental research on aluminium grains at nanoscale level [3].

A significant aspect of integrable systems is its interdisciplinary character, i.e., they can be found in different areas of physics. The Ising and the Heisenberg models [4] in statistical mechanics, the $t-J$ and Hubbard models [5] in condensed matter physics, the nonlinear $\sigma$-model [6] in quantum field theory, the interacting Boson model [7] in nuclear physics and more recently the two-site Bose-Hubbard model [8] in atomic and molecular physics are just some representative examples of the high impact and potentiality of these systems. Let us remark also the emergence of integrable systems in high energy physics, more particularly in gauge theories [9-11] (for a recent review, see [12]), or string theory, through the recent analysis in super-Yang-Mill theories, see e.g. $[13,14]$.

Therefore, new exactly solvable models are highly welcome and constitute the main focus of the present article. In particular we will concentrate on the construction of integrable generalized models in atomic and molecular physics, most of them related to Bose-Einstein condensates (BECs).

The phenomenon of Bose-Einstein condensation, while predicted long ago [15], is currently one of the most active fields in physics, responsible for many of new perspectives on the potential applications of quantum systems. Since the early experimental realizations of BECs using ultracold dilute alkali gases [16], intense efforts have been devoted to the study of new properties of BEC. In recent years the creation of a molecular BEC from an atomic BEC has been obtained by different techniques [17]. The field was further broadened by the achievement of quantum degeneracy in ultracold fermionic gases [18]. These achievements could lead to new scientific investigations that includes coherent atomic lasers, quantum chemistry, the quantum gas with anisotropic dipolar interactions, quantum information, atomtronics, among many others.

In this context, it is natural to expect that exactly solvable models in the BEC scenario may be of relevance, providing some physical insights [19]. Our main purpose here is to employ the integrable systems machinery in its full power, i.e., exploring all possible types of representations of some algebra (we consider, in particular the $g l(N)$ algebra) to enlarge the family of known exactly solvable models in atomic and molecular physics with the aim that potentially new relevant models emerge. Using this machinery some existing models in the BEC scenario, such as the two-site Bose-Hubbard model [8] will be restored as well as new ones will be obtained. A two-coupled BEC model with a field, a two-coupled BEC-model with different types of atoms and a three-coupled BEC model, among others, will be introduced in this general framework. It is worth to mention here that the popular "BEC-transistor" in atomtronics uses a BEC in a triple well [20]. The models are then solved by means of Bethe ansatz methods.

Our paper is organized as follows: in Section 2 we briefly review the general setting of integrable systems and fix notation. In Section 3 we present our general approach and also discuss the different representations of the $g l(N)$ algebra that will be adopted. Section 4 is devoted to the discussion of the different physical models we can get using this construction. In Section 5 the Bethe ansatz equations of the models are derived. We extend these results to the case of the superalgebra $g l(M \mid N)$ in Section 6 and some applications of this formalism, i.e. models which also include fermions are presented in Section 7. Section 8 is devoted to some concluding remarks. 


\section{Generalities}

\subsection{Monodromy and transfer matrices}

We remind here the general setting used in the context of integrable spin chains and more generally in QISM [21-24]. One starts with a so-called 'algebraic' monodromy matrix $T(u)$, which is an $N \times N$ matrix taking values in an algebra $\mathcal{A}$ :

$$
T(u)=\sum_{i, j=1}^{N} T_{i j}(u) E_{i j} \quad \text { with } T_{i j}(u) \in \mathcal{A} ; E_{i j} \in \operatorname{End}\left(\mathbb{C}^{N}\right),
$$

where $E_{i j}$ are the $N \times N$ elementary matrices (with 1 at position $(i, j)$ and 0 elsewhere). In the main part of the present paper, the algebra $\mathcal{A}$ will be the Yangian of $g l(N), Y(N)$, but we will also study super-Yangians.

The monodromy matrix obeys the so-called FRT relation [25]:

$$
R_{12}(u-v) T_{1}(u) T_{2}(v)=T_{2}(v) T_{1}(u) R_{12}(u-v),
$$

where we have used the standard auxiliary space notation, e.g. $T_{1}(u)=T(u) \otimes \mathbb{I}_{N}$, where $\mathbb{I}_{N}$ is the identity matrix and $R_{12}(u)$ is the $R$ matrix of $\mathcal{A}$. The $R$-matrix obeys the Yang-Baxer equation

$$
R_{12}\left(u_{1}-u_{2}\right) R_{13}\left(u_{1}-u_{3}\right) R_{23}\left(u_{2}-u_{3}\right)=R_{23}\left(u_{2}-u_{3}\right) R_{13}\left(u_{1}-u_{3}\right) R_{12}\left(u_{1}-u_{2}\right) .
$$

It will also be unitary

$$
R_{12}\left(u_{1}-u_{2}\right) R_{21}\left(u_{2}-u_{1}\right)=f\left(u_{1}, u_{2}\right) \mathbb{I} \otimes \mathbb{I},
$$

where $f\left(u_{1}, u_{2}\right)$ is some known function.

It is well known that one can produce monodromy matrix for several sites by applying the coproduct $\Delta T(u)=T(u) \otimes T(u) \equiv T^{[1]}(u) T^{[2]}(u)$ where the superscript labels in which copy of the algebra $T(u)$ acts. More generally, one can consider

$$
T(u)=T^{[1]}(u) T^{[2]}(u) \cdots T^{[L]}(u)
$$

as a monodromy matrix. This is the base of spin chain models, the copies of the algebra defining (upon representation) the $L$ quantum spaces (sites) of the chain, see e.g. [26-29] and references therein. In what follows, we will call $T^{[n]}(u)$ the elementary monodromy matrices, the product of all these elementary matrices providing the 'real' monodromy matrix.

Let us stress that to fix a physical model, one has to represent the monodromy matrix, i.e. assign to each of the $T^{[n]}(u)$ a representation of the algebra $\mathcal{A}$ : changing the representations will lead to different physical models.

Once we have a (represented) monodromy matrix $T(u)$, then (2.1) ensures that the transfer matrix $t(u)=\operatorname{tr} T(u)$ obeys

$$
[t(u), t(v)]=0 .
$$

Expanding $t(u)$ in the variable $u$ leads to commuting integral of motions, one of them being the chosen Hamiltonian of the system. For instance, in spin chain models, one takes this Hamiltonian to be $\mathcal{H}=t(0)^{-1} t^{\prime}(0)$, while e.g. in the Bose-Hubbard model, it is simply $t(0)$. The other quantities constructed from the transfer matrix just produce conserved quantities. If the number of such (independent) conserved quantities is sufficiently large, the system is said to be integrable. 


\subsection{Automorphisms of the monodromy matrices}

We present some automorphisms of the relation (2.1) that will be of some use in the physical models we will study.

The first automorphism is built on the transposition: starting from a monodromy matrix $T(u)$, it is easy to show that

$$
T(u) \rightarrow T^{t}(-u) \text { i.e. } \quad T_{i j}(u) \rightarrow T_{j i}(-u),
$$

where the transposition is done in the auxiliary space. The proof relies on the unitary relation (2.3) of the $R$-matrix. We will call this automorphism the sign-transposition.

Another automorphism is the conjugation by a constant matrix

$$
T(u) \rightarrow M T(u) M^{-1} \quad \text { with } M \in \operatorname{End}\left(\mathbb{C}^{N}\right),
$$

which is a consequence of the invariance of the $R$-matrix

$$
M_{1} M_{2} R_{12}(x)=R_{12}(x) M_{1} M_{2} .
$$

We will call this automorphism a conjugation.

A particular case of conjugation is the dilatation automorphism

$$
T(u) \rightarrow \alpha T(u) \quad \text { with } \alpha \in \mathbb{C} .
$$

\subsection{Hermiticity}

The elementary monodromy matrices we will consider below are always Hermitian

$$
(T(u))^{\dagger}=T(u) \quad \text { i.e. } \quad T_{i j}^{\dagger}(u)=T_{j i}(u) .
$$

This implies that

$$
(T(u))^{\dagger}=\left(T^{[1]}(u) T^{[2]}(u)\right)^{\dagger}=T^{[2]}(u) T^{[1]}(u)
$$

so that the total monodromy matrix is not Hermitian. However, using cyclicity of the trace, we get:

$$
t^{\dagger}(u)=\operatorname{tr}\left(T^{[2]}(u) T^{[1]}(u)\right)=\operatorname{tr}\left(T^{[1]}(u) T^{[2]}(u)\right)=t(u) .
$$

Thus, the transfer matrix is Hermitian. This property is valid only when $L=2$ (and $L=1$ ), cyclicity being not sufficient to get Hermiticity of the transfer matrix as soon as $L \geqslant 3$. For this reason, we will focus below on the case $L=2$, hence ensuring Hermitian Hamiltonians. We shall see that even with this restriction, we will get most of the models used in the BEC context, as well as new ones.

\section{Bosonic $g l(N)$ models}

We present here the general approach we use, focusing on the case of the Yangian $Y(N)=$ $Y(g l(N))$ [30]. Other cases are presented in the sections below. The $R$-matrix we consider takes the form $[4,31,32]$ :

$$
R_{12}(x)=\mathbb{I} \otimes \mathbb{I}-\frac{1}{x} P_{12},
$$


where

$$
P_{12}=\sum_{i, j=1}^{N} E_{i j} \otimes E_{j i}
$$

is the permutation operator.

\subsection{Conserved quantities}

Since $L=2$, the monodromy and transfer matrices have an expansion

$$
\begin{aligned}
& T_{k l}(u)=\sum_{n=0}^{2} u^{n} T_{k l}^{(n)} \quad \text { with } T_{k l}^{(2)}=\omega_{k} \delta_{k l} \text { and } \omega_{k} \in \mathbb{C}, \\
& t(u)=t_{2} u^{2}+t_{1} u+t_{0} \quad \text { with } t_{2} \in \mathbb{C} .
\end{aligned}
$$

For BEC models, one generally uses $t_{0}=t(0)$ as an Hamiltonian, while $t_{1}$ and $t_{2}$ correspond to integrals of motion. It is easy to see that $t_{2}$ is just a number, but from the explicit form of the $R$-matrix, one can get other conserved quantities.

Indeed starting from the relation (2.1) and projecting on the basis elements $E_{i j} \otimes E_{k l}$ in the auxiliary spaces, one gets:

$$
\left[T_{i j}(u), T_{k l}(v)\right]=\frac{1}{u-v}\left(T_{k j}(u) T_{i l}(v)-T_{k j}(v) T_{i l}(u)\right) .
$$

Then, taking $i=j$, summing on $j$, and looking at the coefficient of $v$, one gets

$$
\left[t(u), T_{k l}^{(1)}\right]=\left(\omega_{k}-\omega_{l}\right)\left(T_{k l}^{(0)}+u T_{k l}^{(1)}\right) .
$$

This proves in particular that the quantities

$$
I_{k}=T_{k k}^{(1)} \quad \forall k=1, \ldots, N,
$$

commute with the transfer matrix and are in involution. Thus, they generate integrals of motions.

Let us remark that, following the value of the $\omega_{k}$ parameters, one could get more conserved quantities (through the $T_{k l}^{(1)}, k \neq l$, generators), but they will not form an Abelian subalgebra: they will generate a symmetry algebra for the model.

\subsection{Spin chain monodromy matrices}

The elementary monodromy matrices for the Yangian can be specialized to $g l(N)$ monodromy matrices using the so-called evaluation map. This amounts to take these elementary monodromy matrices to be of the form

$$
L(u)=\sum_{i, j=1}^{N} L_{i j}(u) E_{i j} \quad \text { with } L_{i j}(u)=u \delta_{i j}+e_{i j},
$$

or in matricial form

$$
L(u)=\left(\begin{array}{ccccc}
u+e_{11} & e_{12} & e_{13} & \cdots & e_{1 N} \\
e_{21} & u+e_{22} & e_{23} & \cdots & e_{2 N} \\
\vdots & \ddots & \ddots & \ddots & \vdots \\
e_{N-1,1} & & & & e_{N-1, N} \\
e_{N 1} & e_{N 2} & \cdots & e_{N, N-1} & u+e_{N N}
\end{array}\right) .
$$


Here, $e_{i j}$ are $g l(N)$ unrepresented generators obeying

$$
\left[e_{i j}, e_{k l}\right]=\delta_{j k} e_{i l}-\delta_{i l} e_{k j} .
$$

It is easy to show that $L(u)$ obey the relation (2.1) with the $R$-matrix (3.1). Hermiticity of $L(u)$ is ensured by $e_{i j}^{\dagger}=e_{j i}$. Moreover, one can use the Yangian shift automorphism $u \rightarrow u+w$ to get extra free parameters. Thus,

$$
T(u)=L^{[1]}\left(u+w_{1}\right) L^{[2]}\left(u+w_{2}\right)
$$

leads to Hermitian integrable models with transfer matrix

$$
t(u)=\sum_{j=1}^{N}\left(u+w_{1}+e_{j j}^{[1]}\right) \otimes\left(u+w_{2}+e_{j j}^{[2]}\right)+\sum_{j \neq k}^{N} e_{j k}^{[1]} \otimes e_{k j}^{[2]} .
$$

In the context of spin chain models, the parameters $w_{j}$ are called inhomogeneity parameters.

As already stated, it is the choice of a $g l(N)$ representation for each of the sites that will determine the physical model one wishes to work on. When the representations are highest weight finite dimensional ones, it leads to spin chains models. They have been extensively studied and we just repeat here well-known facts to illustrate the techniques we shall use with different representations.

For instance, one can take the fundamental representation of $g l(N)$

$$
\pi\left(e_{i j}\right)=E_{i j}, \quad i, j=1, \ldots, N,
$$

for both elementary monodromy matrices, leading to a (well-known and somehow trivial) twosite spin chain. Specifying furthermore to the case of $g l(2)$, one recovers the Pauli matrices

$$
\pi\left(e_{12}\right)=\sigma_{+} ; \quad \pi\left(e_{21}\right)=\sigma_{-} ; \quad \pi\left(e_{11}-e_{22}\right)=\sigma_{z} ; \quad \pi\left(e_{11}+e_{22}\right)=\mathbb{I}_{2}
$$

leading to a Hamiltonian

$$
H=t(0)=\sigma_{+} \otimes \sigma_{-}+\sigma_{-} \otimes \sigma_{+}+\frac{1}{2} \sigma_{z} \otimes \sigma_{z}+\frac{1}{2} .
$$

Of course, one could choose another representation, for instance, for $g l(2)$, take the spin 1 representation

$$
\begin{aligned}
& \pi\left(e_{12}\right)=\left(\begin{array}{ccc}
0 & 1 & 0 \\
0 & 0 & 1 \\
0 & 0 & 0
\end{array}\right)=S_{+} ; \quad \pi\left(e_{21}\right)=\left(\begin{array}{lll}
0 & 0 & 0 \\
1 & 0 & 0 \\
0 & 1 & 0
\end{array}\right)=S_{-} ; \\
& S_{z}=\left(\begin{array}{ccc}
1 & 0 & 0 \\
0 & 0 & 0 \\
0 & 0 & -1
\end{array}\right) ; \\
& \pi\left(e_{11}\right)=\left(\begin{array}{ccc}
1 & 0 & 0 \\
0 & \frac{1}{2} & 0 \\
0 & 0 & 0
\end{array}\right)=\frac{1}{2}\left(S_{z}+\mathbb{I}_{3}\right) ; \quad \pi\left(e_{22}\right)=\left(\begin{array}{lll}
0 & 0 & 0 \\
0 & \frac{1}{2} & 0 \\
0 & 0 & 1
\end{array}\right)=\frac{1}{2}\left(\mathbb{I}_{3}-S_{z}\right)
\end{aligned}
$$

leading to the Hamiltonian

$$
H=t(0)=S_{+} \otimes S_{-}+S_{-} \otimes S_{+}+\frac{1}{2} S_{z} \otimes S_{z}+\frac{1}{2} .
$$

Note that in both cases, the parameters $w_{n}$ do not play any role because the spin chain is too simple. For the same reason, the above Hamiltonians coincide with $t(0)^{-1} t^{\prime}(0)$. 


\subsection{Oscillator monodromy matrices}

The above framework can be generalized to other (infinite dimensional) representations of $g l(N)$.

\subsubsection{Bosonic and fermionic representations of $g l(N)$} tions

We start with $N$ couples of creation/annihilation operators $\left(a_{i}, a_{i}^{\dagger}\right)$, with commutation rela-

$$
\left[a_{i}, a_{j}^{\dagger}\right]=\mu_{i} \delta_{i j} ; \quad\left[a_{i}^{\dagger}, a_{j}^{\dagger}\right]=\left[a_{i}, a_{j}\right]=0 .
$$

From these relations, it is straightforward to check that $\mathcal{L}(u)$ defined by

$$
\mathcal{L}(u)=\sum_{i, j=1}^{N} \mathcal{L}_{i j}(u) E_{i j} \quad \text { with } \mathcal{L}_{i j}(u)=\mu_{i} u \delta_{i j}+\frac{q_{i}}{q_{j}} a_{i}^{\dagger} a_{j},
$$

obey the relations

$$
\left[\mathcal{L}_{i j}(u), \mathcal{L}_{k l}(v)\right]=\frac{1}{u-v}\left(\mathcal{L}_{k j}(u) \mathcal{L}_{i l}(v)-\mathcal{L}_{k j}(v) \mathcal{L}_{i l}(u)\right)
$$

It is equivalent to

$$
R_{12}(u-v) \mathcal{L}_{1}(u) \mathcal{L}_{2}(v)=\mathcal{L}_{2}(v) \mathcal{L}_{1}(u) R_{12}(u-v) \quad \text { with } R_{12}(x)=\mathbb{I} \otimes \mathbb{I}-\frac{1}{x} P_{12},
$$

so that

$$
T(u)=\mathcal{L}^{[1]}\left(u+w_{1}\right) \mathcal{L}^{[2]}\left(u+w_{2}\right)
$$

provides an integrable model.

In fact, this calculation is valid for an arbitrary number of sites, ${ }^{1}$ and it just corresponds to a choice of (infinite dimensional) $g l(N)$ representation

$$
\pi\left(e_{i j}\right)=a_{i}^{\dagger} a_{j}, \quad i, j=1, \ldots, N,
$$

for the elementary monodromy matrices (3.7). The highest weight is the Fock space vacuum $|0\rangle$, but the representation is reducible and is an infinite sum of finite dimensional representations with fixed 'particle number' $\mathcal{N}=\sum_{i} a_{i}^{\dagger} a_{i}$. We will call the corresponding monodromy matrix an 'homogeneous oscillator monodromy matrix'.

Focusing on Hermitian elementary matrices, one is led to

$$
\mu_{j} \in \mathbb{R} \quad \text { and } \quad\left|q_{i}\right|^{2}=\left|q_{j}\right|^{2} \quad \forall i, j .
$$

The last equation imposes

$$
q_{j}=q_{0} e^{i \theta_{j}} \quad \text { with } q_{0}, \theta_{j} \in \mathbb{R} .
$$

The parameter $q_{0}$ is irrelevant for $\mathcal{L}(u)$, and since

$$
a_{j} \rightarrow e^{i \theta_{j}} a_{j} \quad \text { and } \quad a_{j}^{\dagger} \rightarrow e^{-i \theta_{j}} a_{j}^{\dagger}
$$

\footnotetext{
1 Of course, we will potentially lose Hermiticity of the transfer matrix when $L \geqslant 3$.
} 
is an invariance of the algebra, one can restrict to the case

$$
\mathcal{L}_{i j}(u)=\mu_{i} u \delta_{i j}+a_{i}^{\dagger} a_{j} \quad \text { with } \mu_{j} \in \mathbb{R},
$$

or in matricial form

$$
\mathcal{L}(u)=\left(\begin{array}{ccccc}
\mu_{1} u+n_{1} & a_{1}^{\dagger} a_{2} & a_{1}^{\dagger} a_{3} & \ldots & a_{1}^{\dagger} a_{N} \\
a_{2}^{\dagger} a_{1} & \mu_{2} u+n_{2} & a_{2}^{\dagger} a_{3} & \ldots & a_{2}^{\dagger} a_{N} \\
\vdots & \ddots & \ddots & \ddots & \vdots \\
a_{N-1}^{\dagger} a_{1} & & & & a_{N-1}^{\dagger} a_{N} \\
a_{N}^{\dagger} a_{1} & a_{N}^{\dagger} a_{2} & \ldots & a_{N}^{\dagger} a_{N-1} & \mu_{N} u+n_{N}
\end{array}\right) \quad \text { with } n_{i}=a_{i}^{\dagger} a_{i}
$$

In general, one takes the values $\mu_{j}=1$ to get canonical commutation relations. Then, $\mathcal{L}(u)$ has a leading term (in $u$ ) which is just the identity matrix, in accordance with the definition of the Yangian matrix $L(u)$.

Let us remark that there exists also a fermionic $g l(N)$ representation where now $a_{j}$ and $a_{j}^{\dagger}$ are fermionic operators:

$$
\mathcal{L}_{i j}^{f}(u)=\mu_{i} u \delta_{i j}+a_{i}^{\dagger} a_{j} \quad \text { with } \mu_{j} \in \mathbb{R} .
$$

In that case, the representation is finite dimensional, since the oscillators now obey the supplementary relations $\left(a_{j}\right)^{2}=0=\left(a_{j}^{\dagger}\right)^{2}$. This possibility will be used to produce some fermionic models.

\subsubsection{Inhomogeneous oscillator monodromy matrices}

The above calculation is valid whatever the values of the numbers $\mu_{i}$ are. In particular, one can take the value $\mu_{j}=0$, for some $j \in J \subset[1, N]$ : the corresponding $\mathcal{L}(u)$ matrix will still obey (2.1) with the $R$-matrix (3.1). This particular value $\mu_{j}=0$ allows a (scalar) representation $a_{j}=\alpha_{j} \in \mathbb{C}$ and $a_{j}^{\dagger}=\alpha_{j}^{*}, j \in J$, of the oscillator algebra. For obvious reason, we will call these operators 'constant oscillators', and 'inhomogeneous oscillator monodromy matrix' the corresponding elementary monodromy matrix. To distinguish it from the homogeneous one, when needed, we will denote it as $\Lambda(u)$ instead of $\mathcal{L}(u)$.

Hence, $\Lambda(u)$ is an $N \times N$ matrix built on $p=N-|J|$ couples $\left(a_{j}, a_{j}^{\dagger}\right), p$ being independent from $N$ (provided it is smaller than $N$ ). Then, $T(u)$ will lead to a transfer matrix based $p_{1}+p_{2}$ oscillators, where $p_{n}$ is the number of oscillators in $T^{[n]}(u) \equiv \Lambda^{[n]}(u), n=1,2$.

Let us remark that since we have taken the limit $\mu_{j} \rightarrow 0$ for $j \in J$, the elementary monodromy matrices do not start with $\mathbb{I}_{N}$, but rather with a non-invertible diagonal matrix. In that sense, we are not in the Yangian context anymore. However, since the relation (2.1) is still obeyed with the $R$-matrix (3.1), this does not affect the relation (2.4), so we are still in the framework of Hermitian integrable models. The underlying algebraic structure, which is very close to the Yangian, was studied in [33] and is called 'truncated Yangians'. Keeping in mind this restriction, we will lose keep writing that we are in the Yangian context.

Note also that the limit $\mu_{j} \rightarrow 0$ (and $a_{j}, a_{j}^{\dagger}$ constant) can be taken at the very end of the calculations. Hence, we can consider a general $\mathcal{L}(u)$ matrix, keeping in mind that, to get a $\Lambda(u)$ elementary monodromy matrix, and depending on the model one wishes to study, some of the $a_{j}$, $a_{j}^{\dagger}$ operators will be in fact complex numbers $\alpha_{j}, \alpha_{j}^{*}$, and the corresponding $\mu_{j}=0$. For other oscillators, one chooses in general $\mu= \pm 1$. 
Finally, let us remark that for the fermionic $g l(N)$ representation, since now the $a_{j}$ and $a_{j}^{\dagger}$ operators obey the supplementary relations $\left(a_{j}\right)^{2}=0=\left(a_{j}^{\dagger}\right)^{2}$, it is not possible to take them as non-vanishing constants. Taking all the constants to be zero leads to trivial models, hence, fermions are excluded from $\Lambda(u)$ when dealing with $Y(N)$. Fortunately, we will see below that one can recover them when studying models based on super-Yangians (see Section 6).

\subsubsection{Automorphisms of oscillator algebra}

An automorphism will be used to produce new terms in the Hamiltonians. It exists only for the bosonic algebra, and consists in a shift by a constant:

$$
\left(a, a^{\dagger}, \mu\right) \rightarrow\left(a+\alpha, a^{\dagger}+\alpha^{*}, \mu\right), \quad \alpha \in \mathbb{C} \quad \text { for bosons. }
$$

We will call this automorphism a shift of the oscillator algebra. It can be used to produce boundary terms in the different models.

\section{4. $g l(N)$ transfer matrices}

We have seen that we have essentially two types of elementary matrices at our disposal, the matrices $L(u)$ and $\mathcal{L}(u)$, so that one gets three types of transfer matrix:

$$
\begin{aligned}
t(u) & =\operatorname{tr}\left(L^{[1]}\left(u+w_{1}\right) L^{[2]}\left(u+w_{2}\right)\right) \\
& \sim u \sum_{i=1}^{N}\left(E_{i i}^{[1]}+E_{i i}^{[2]}\right)+\sum_{i, j=1}^{N} E_{i j}^{[1]} E_{j i}^{[2]}+\sum_{i=1}^{N}\left(w_{2} E_{i i}^{[1]}+w_{1} E_{i i}^{[2]}\right), \\
t(u) & =\operatorname{tr}\left(L^{[1]}\left(u+w_{1}\right) \mathcal{L}^{[2]}\left(u+w_{2}\right)\right) \\
& \sim u \sum_{i=1}^{N}\left(\mu_{i} E_{i i}^{[1]}+n_{i}\right)+\sum_{i, j=1}^{N} E_{i j}^{[1]} a_{j}^{\dagger} a_{i}+\sum_{i=1}^{N}\left(w_{2} E_{i i}^{[1]}+w_{1} n_{i}\right), \\
t(u) & =\operatorname{tr}\left(\mathcal{L}^{[1]}\left(u+w_{1}\right) \mathcal{L}^{[2]}\left(u+w_{2}\right)\right) \\
& \sim u \sum_{i=1}^{N}\left(\mu_{i} n_{b i}+v_{i} n_{a i}\right)+\sum_{i, j=1}^{N} b_{i}^{\dagger} a_{j}^{\dagger} a_{i} b_{j}+\sum_{i=1}^{N}\left(w_{1} n_{b i}+w_{2} n_{a i}\right),
\end{aligned}
$$

where the $\sim$ sign means equality modulo polynomials in $u$ with constant coefficients. As a notation, we have introduced $E_{i j}^{[n]}=\pi_{n}\left(e_{i j}\right), n=1,2$, the representation of the $g l(N)$ generators in $L^{[n]}(u)$ and called $a_{j}, a_{j}^{\dagger}, \mu_{j}$ (respectively $b_{j}, b_{j}^{\dagger}, v_{j}$ ) the oscillator algebras in $\mathcal{L}^{[1]}(u)$ (respectively in $\mathcal{L}^{[2]}(u)$ ); $n_{a j}$ (respectively $n_{b j}$ ) are the corresponding number operators.

The first transfer matrix is just a two-site spin chain, where the first site carries the 'spin' $\pi_{1}$ of $g l(N)$, and the second site the 'spin' $\pi_{2}$. These models (and their generalization to an arbitrary number of sites) have been studied for a long time, and we will not consider them here. The two other transfer matrices lead to several physical models, depending on the choices of:

- The $g l(N)$ algebra one considers (i.e. the choice of $N)$.

- The $g l(N)$ representation in the $L(u)$ part.

- The characteristic (bosonic or fermionic) of the oscillators in the $\mathcal{L}(u)$ part.

- The number of 'constant oscillators' in the $\Lambda(u) \operatorname{part}(\mathrm{s})$. 
- The values (specially zero or not) of the parameters corresponding to these 'constant oscillators'.

- The use or not of the different automorphisms.

The next section is devoted to the presentation of the different physical models one can get from these choices.

\section{Examples of $g l(N)$ BEC models}

For simplicity, we now focus on the case where the parameters involved in the elementary matrices are real, and normalize the commutator of the oscillators to 1. More general Hamiltonians (still Hermitian) can be obtained keeping complex parameters, as detailed above.

\subsection{Models based on two by two matrices}

The possible elementary monodromy matrices take the form

$$
\begin{aligned}
& L(u)=\left(\begin{array}{cc}
u+\frac{1}{2} S_{z} & S_{+} \\
S_{-} & u-\frac{1}{2} S_{z}
\end{array}\right), \quad \quad \mathcal{L}(u)=\left(\begin{array}{cc}
u+n_{1} & a_{1}^{\dagger} a_{2} \\
a_{2}^{\dagger} a_{1} & u+n_{2}
\end{array}\right), \\
& \Lambda(u)=\left(\begin{array}{cc}
u+n & \beta a^{\dagger} \\
\beta a & \beta^{2}
\end{array}\right), \quad \hat{\Lambda}(u)=\left(\begin{array}{cc}
-\beta^{2} & \beta a^{\dagger} \\
\beta a & u-n
\end{array}\right)
\end{aligned}
$$

plus possibly the use of shift automorphisms. We have used the sign-transposition and dilation automorphisms

$$
\hat{\Lambda}(u) \rightarrow-\hat{\Lambda}^{t}(-u)
$$

plus a redefinition of the $\beta$ parameter to make $\hat{\Lambda}(u)$ similar to $\Lambda(u)$.

Apart from the spin chain model presented in Section 3.2, one gets 5 different models. The conserved quantities $I_{j}$ have the general form:

$$
I_{1}=\mu_{b 1} n_{a 1}+\mu_{a 1} n_{b 1} \quad \text { and } \quad I_{2}=\mu_{b 2} n_{a 2}+\mu_{a 2} n_{b 2},
$$

where $\left(a_{1}, a_{2}\right)$ refer to the first elementary monodromy matrix, and $\left(b_{1}, b_{2}\right)$ to the second one.

\subsubsection{A spin-boson model}

We consider $t(u)=\operatorname{tr} L\left(u+w_{1}\right) \Lambda\left(u+w_{2}\right)$. Up to irrelevant constant terms, it takes the form

$$
t(u) \equiv u\left(\frac{1}{2} S_{z}+n\right)+w_{1}\left(\frac{1}{2} S_{z}+n\right)+\left(\alpha S_{+} a+\alpha S_{-} a^{\dagger}\right)+\frac{1}{2} S_{z} n
$$

leading to Hamiltonian $H=t(0)$ with conserved quantity

$$
I=n+\frac{1}{2} S_{z}
$$

Above $a^{\dagger}$ (a) denotes the single-mode field creation (annihilation) operator, $S_{z}, S_{ \pm}$the atomic inversion, rising and lowering operators and $w_{1}$ is the transition frequency. This model describes the interaction of a two-level atom with a single-mode radiation field. It was derived in [34,35] using different methods and it reduces to the Jaynes-Cummings Hamiltonian on resonance and in the rotating-wave approximation in the absence of the last term [36]. Despite of its simplicity, this 
model has been a source of insight into a better comprehension of the nuances of the interaction between light and matter. It is important to remark that a Jaynes-Cummings model interaction can be experimentally realized in cavity-QED setups and also, as an effective interaction in laser cooled trapped ions [37].

Again, the shift automorphism produces boundary term

$$
H_{\text {bound }}=\beta\left(\frac{1}{2} S_{z}\left(a^{\dagger}+a\right)+\alpha\left(S_{+}+S_{-}\right)+w_{1}\left(a^{\dagger}+a\right)\right)
$$

to the transfer matrix. The conserved quantity is modified to

$$
I^{\prime}=n+\frac{1}{2} S_{z}+\beta\left(a^{\dagger}+a\right)
$$

\subsubsection{Generalized spin-boson model}

We consider $t(u)=\operatorname{tr} L\left(u+w_{1}\right) \mathcal{L}\left(u+w_{2}\right)$. Up to irrelevant constant terms, it takes the form

$$
t(u) \equiv u\left(n_{1}+n_{2}\right)+\frac{1}{2} S_{z}\left(n_{1}-n_{2}\right)+S_{+} a_{1}^{\dagger} a_{2}+S_{-} a_{2}^{\dagger} a_{1}+w_{1}\left(n_{1}+n_{2}\right)
$$

leading to Hamiltonian

$$
H=t(0)=\frac{1}{2} S_{z}\left(n_{1}-n_{2}\right)+S_{+} a_{1}^{\dagger} a_{2}+S_{-} a_{2}^{\dagger} a_{1}+w_{1}\left(n_{1}+n_{2}\right)
$$

with conserved quantities

$$
I_{1}=n_{1}+\frac{1}{2} S_{z} ; \quad I_{2}=n_{2}-\frac{1}{2} S_{z}
$$

Above the oscillators $a_{j}, j=1,2$, denote two radiation fields (two photons, for example) interacting with a two-level atom. The atom-field interacting term could be interpreted as a scattering of two fields with a two-level atom. Here we mention that if linearly polarized light is used, it is possible to have the same transition frequency $w_{1}$ (see, for example [38]).

When the oscillators are bosonic, one can use the shift automorphism to add a boundary term:

$$
\begin{aligned}
H_{\text {bound }}= & \alpha_{1}\left(\frac{1}{2} S_{z}\left(a_{1}^{\dagger}+a_{1}+\alpha_{1}\right)+S_{+} a_{2}+S_{-} a_{2}^{\dagger}+w_{1}\left(a_{1}^{\dagger}+a_{1}\right)\right) \\
& +\alpha_{2}\left(\frac{1}{2} S_{z}\left(a_{2}^{\dagger}+a_{2}+\alpha_{2}\right)+S_{+} a_{1}+S_{-} a_{1}^{\dagger}+w_{1}\left(a_{2}^{\dagger}+a_{2}\right)\right)+\alpha_{1} \alpha_{2}\left(S_{+}+S_{-}\right)
\end{aligned}
$$

to the transfer matrix. The conserved quantities then become

$$
\begin{aligned}
& I_{1}^{\prime}=n_{1}+\frac{1}{2} S_{z}+\alpha_{1}\left(a_{1}^{\dagger}+a_{1}\right), \\
& I_{2}^{\prime}=n_{2}-\frac{1}{2} S_{z}+\alpha_{2}\left(a_{2}^{\dagger}+a_{2}\right) .
\end{aligned}
$$




\subsubsection{Simple heteroatomic-molecular BEC model}

We consider $t(u)=\operatorname{tr} \mathcal{L}^{[1]}\left(u+w_{1}\right) \mathcal{L}^{[2]}\left(u+w_{2}\right)$, with $a_{j}, a_{j}^{\dagger}, j=1,2$, for $\mathcal{L}^{[1]}$ and $b_{j}, b_{j}^{\dagger}$, $j=1,2$, for $\mathcal{L}^{[2]}$.

Up to irrelevant constant terms, the transfer matrix takes the form

$$
t(u) \sim u\left(n_{1}+n_{2}\right)+n_{a 1} n_{b 1}+n_{a 2} n_{b 2}+a_{1}^{\dagger} b_{2}^{\dagger} b_{1} a_{2}+a_{2}^{\dagger} b_{1}^{\dagger} b_{2} a_{1}+w_{1} n_{b}+w_{2} n_{a},
$$

where we have introduced the notation

$$
\begin{array}{ll}
n_{a 1}=a_{1}^{\dagger} a_{1} ; \quad & n_{b 1}=b_{1}^{\dagger} b_{1} ; \quad n_{a 2}=a_{2}^{\dagger} a_{2} ; \quad n_{b 2}=b_{2}^{\dagger} b_{2} ; \\
n_{1}=n_{a 1}+n_{b 1} ; & n_{2}=n_{a 2}+n_{b 2} ; \\
n_{a}=n_{a 1}+n_{a 2} ; & n_{b}=n_{b 1}+n_{b 2} .
\end{array}
$$

It leads to Hamiltonian $H=t(0)$ with conserved quantities

$$
I_{1}=n_{1} ; \quad I_{2}=n_{2} .
$$

This Hamiltonian is a particular case of the one presented in Section 4.1.4, and we postpone the physical discussion to this section.

Finally, using the quantum determinant (see Appendix A), one can also show from $c_{2}$ given in appendix that $n_{a} n_{b}$ is also conserved, so that one finally gets as conserved quantities

$$
n_{1}=n_{a 1}+n_{b 1} ; \quad n_{2}=n_{a 2}+n_{b 2} ; \quad n_{a}=n_{a 1}+n_{a 2} ; \quad n_{b}=n_{b 1}+n_{b 2}
$$

three of them being independent.

\subsubsection{Heteroatomic-molecular BEC model}

We consider $t(u)=\operatorname{tr} \mathcal{L}\left(u+w_{1}\right) \Lambda\left(u+w_{2}\right)$, with $a, a^{\dagger}$ and $b, b^{\dagger}$ for $\mathcal{L}(u)$ and $c, c^{\dagger}$ for $\Lambda(u)$.

Up to irrelevant constant terms, it takes the form

$$
t(u) \equiv u\left(n_{a}+n_{c}\right)+n_{a} n_{c}+\beta^{2} n_{b}+\beta\left(b^{\dagger} c^{\dagger} a+a^{\dagger} b c\right)+w_{1} n_{c}+w_{2} n_{a} .
$$

It leads to Hamiltonian $t(0)$ with conserved quantities

$$
I_{1}=n_{a}+n_{b} ; \quad I_{2}=n_{a}+n_{c} .
$$

It is trivial to check that this Hamiltonian (adding terms $I_{1}^{2}$ and $I_{2}^{2}$ ) corresponds to the heteroatomic-molecular Bose-Einstein condensate model $[39,40]$

$$
\begin{aligned}
\mathcal{H}= & U_{a a} n_{a}^{2}+U_{b b} n_{b}^{2}+U_{c c} n_{c}^{2}+U_{a b} n_{a} n_{b}+U_{a c} n_{a} n_{c}+U_{b c} n_{b} n_{c} \\
& +\mu_{a} n_{a}+\mu_{b} n_{b}+\mu_{c} n_{c}+\Omega\left(a^{\dagger} b c+c^{\dagger} b^{\dagger} a\right),
\end{aligned}
$$

for the particular choice of the couplings $\frac{1}{2} U_{a a}=U_{b b}=U_{c c}=U_{0}+U_{1}, U_{a b}=4 U_{0}, U_{b c}=0$, $U_{a c}=4 U_{1}+1, \mu_{a}=w_{2}, \mu_{b}=\beta^{2}, \mu_{c}=w_{1}, \Omega=\beta$.

In this context, the parameters $U_{i j}$ describe $S$-wave scattering, $\mu_{i}$ are external potentials and $\Omega$ is the amplitude for interconversion of atoms and molecules. One gets a three-mode Hamiltonian describing a Bose-Einstein condensate with two distinct species of atoms, denoted $b$ and $c$, which can combine to produce a molecule $a$ [39]. The total atom number $\left(I_{1}+I_{2}\right)$ and the imbalance between the atomic modes $\left(I_{1}-I_{2}\right)$ are conserved quantities. A detailed classical and quantum analysis of this model reveals unexpected scenarios, such as the emergence of quantum phases when the imbalance is zero [41]. 


\subsubsection{The two-site Bose-Hubbard model}

We consider $t(u)=\operatorname{tr} \Lambda^{[1]}\left(u+w_{1}\right) \Lambda^{[2]}\left(u+w_{2}\right)$, with $a, a^{\dagger}$ for $\Lambda^{[1]}$ and $b, b^{\dagger}$ for $\Lambda^{[2]}$.

Up to irrelevant constant terms, it takes the form

$$
t(u) \equiv u\left(n_{a}+n_{b}\right)+n_{a} n_{b}+\omega\left(b^{\dagger} a+a^{\dagger} b\right)+w_{1} n_{b}+w_{2} n_{a}
$$

with $w=\alpha \beta$. It leads to Hamiltonian $t(0)$ with conserved quantity

$$
I=n_{a}+n_{b} .
$$

It is easy to verify that by combining the conserved quantity $I$ with the Hamiltonian $t(0)$ and choosing properly the coupling constants, we arrive $\mathrm{at}^{2}$

$$
\mathcal{H}=\frac{K}{8}\left(n_{a}-n_{b}\right)^{2}-\frac{\Delta \mu}{2}\left(n_{a}-n_{b}\right)-\frac{\mathcal{E}_{\mathcal{J}}}{2}\left(a^{\dagger} b+b^{\dagger} a\right) .
$$

This is the two-site Bose-Hubbard model, also known as the canonical Josephson Hamiltonian [8]. It describes the tunneling between two single particle states or modes $(a$ and $b$ ), which can be separated spatially (two wells) or internally (two different internal quantum numbers). The parameter $K$ corresponds to the atom-atom interaction, $\Delta \mu$ is the external potential and $\mathcal{E}_{J}$ is the coupling for the tunneling. Its exact solution in some asymptotic limits was presented in [42]. Despite of its apparent simplicity, this model predicts the existence of a threshold coupling between a delocalized and self-trapped phase $[43,44]$, in qualitative agreement with experiments [45].

\subsection{Models based on three by three matrices}

The number of possibilities increases very fast, we present here only some cases that we found physically relevant. The interested reader can easily compute the other models using the techniques we have described.

\subsubsection{Two-coupled BEC model with a single-mode field}

We consider the elementary monodromy matrices

$$
\begin{aligned}
& \Lambda^{[1]}(u)=\left(\begin{array}{ccc}
u+n_{a} & a^{\dagger} b & \alpha_{3} a^{\dagger} \\
b^{\dagger} a & u+n_{b} & \alpha_{3} b^{\dagger} \\
\alpha_{3} a & \alpha_{3} b & \alpha_{3}^{2}
\end{array}\right) \text { and } \Lambda^{[2]}(u)=\left(\begin{array}{ccc}
u+n_{c} & \beta_{1} c^{\dagger} & \beta_{2} c^{\dagger} \\
\beta_{1} c & \beta_{1}^{2} & \beta_{1} \beta_{2} \\
\beta_{2} c & \beta_{1} \beta_{2} & \beta_{2}^{2}
\end{array}\right), \\
& T(u)=\Lambda^{[1]}\left(u+w_{1}\right) \Lambda^{[2]}\left(u+w_{2}\right) .
\end{aligned}
$$

Then, the transfer matrix reads

$$
\begin{aligned}
t(u) \sim & \left(n_{c}+n_{a}\right) u+\beta_{1}^{2} n_{b}+n_{a} n_{c}+w_{1} n_{c}+w_{2} n_{a}+\alpha_{3} \beta_{2}\left(a^{\dagger} c+c^{\dagger} a\right) \\
& +\beta_{1}\left(a^{\dagger} b c+b^{\dagger} c^{\dagger} a\right)+\alpha_{3} \beta_{1} \beta_{2}\left(b^{\dagger}+b\right)
\end{aligned}
$$

with conserved quantity

$$
I=n_{a}+n_{c}
$$

\footnotetext{
2 More specifically, we have $H=c\left(I^{2}-4 t(0)\right)$, where the following identification has been done: $K / 8=c ;(\Delta \mu) / 2=$ $4 c w_{2}=-4 c w_{1} ; \mathcal{E}_{\mathcal{J}} / 2=4 c w$.
} 
This leads to the Hamiltonian ${ }^{3}$

$$
\mathcal{H}=\mu_{a} n_{a}+\mu_{c} n_{c}+w^{2} n_{b}+\Omega\left(a^{\dagger} c+c^{\dagger} a\right)+w\left(a^{\dagger} b c+c^{\dagger} b^{\dagger} a\right)+\Omega w\left(b^{\dagger}+b\right)
$$

which describes two wells ( $A$ and $C$ ) with atoms interacting with a single-mode field. Above $b^{\dagger}$ and $b$ denote the single field creation and annihilation operators, respectively and $a^{\dagger}$ and $a$ $\left(c^{\dagger}\right.$ and $c$ ) the creation and annihilation operators for a particle in the well $A(C)$. The total number of particles $n=n_{a}+n_{c}$, where $n_{a}=a^{\dagger} a$ and $n_{c}=c^{\dagger} c$ is conserved. The parameter $\Omega$ is the coupling for the tunneling between the two wells, $w$ is the radiation field frequency and $\mu_{a}, \mu_{c}$ are the external potentials. Here there are basically two mechanisms that allow the atoms to trap between the wells: (i) the tunneling effect, which is related with the depth of the wells; (ii) a tunneling which occurs mediated by a single-mode field $b$.

When $w=0$, one has a supplementary relation

$$
\left[t(u), n_{b}\right]=0,
$$

and this Hamiltonian reduces to the two-site Bose-Hubbard (Section 4.1.5). When $\Omega=0$ we get

$$
\left[t(u), n_{b}-n_{c}\right]=0
$$

and this Hamiltonian reduces to that of or that discussed in Section 4.1.4.

\subsubsection{Three coupled BEC model}

We consider the elementary monodromy matrices

$$
\begin{aligned}
& \Lambda^{[1]}(u)=\left(\begin{array}{ccc}
u+n_{1} & a_{1}^{\dagger} a_{2} & \beta_{3} a_{1}^{\dagger} \\
a_{2}^{\dagger} a_{1} & u+n_{2} & \beta_{3} a_{2}^{\dagger} \\
\beta_{3} a_{1} & \beta_{3} a_{2} & \beta_{3}^{2}
\end{array}\right) \text { and } \Lambda^{[2]}(u)=\left(\begin{array}{ccc}
-\beta_{1}^{2} & \beta_{1} \beta_{2} & \beta_{1} a_{3}^{\dagger} \\
\beta_{1} \beta_{2} & -\beta_{2}^{2} & \beta_{2} a_{3}^{\dagger} \\
\beta_{1} a_{3} & \beta_{2} a_{3} & u-n_{3}
\end{array}\right), \\
& T(u)=\Lambda^{[1]}\left(u+w_{1}\right) \Lambda^{[2]}\left(u+w_{2}\right),
\end{aligned}
$$

where in $\Lambda^{[2]}(u)$ we have used the sign-transposition and dilatation automorphisms, and a redefinition of the parameters $\beta_{1}$ and $\beta_{2}$. Then, the transfer matrix reads

$$
\begin{aligned}
t(u) \sim & \beta_{1} \beta_{2}\left(a_{1}^{\dagger} a_{2}+a_{2}^{\dagger} a_{1}\right)+\beta_{3} \beta_{1}\left(a_{1}^{\dagger} a_{3}+a_{3}^{\dagger} a_{1}\right)+\beta_{3} \beta_{2}\left(a_{2}^{\dagger} a_{3}+a_{3}^{\dagger} a_{2}\right) \\
& -\beta_{1}^{2} n_{1}-\beta_{2}^{2} n_{2}-\beta_{3}^{2} n_{3} .
\end{aligned}
$$

It leads to the Hamiltonian $t(0)$ with conserved quantity $I=n_{1}+n_{2}+n_{3}$. This corresponds to a special three coupled BEC model with asymmetric tunneling and external potentials. To our knowledge, this is the first integrable quantum model describing a three-well system. ${ }^{4} \mathrm{We}$ remark here that there has been recently an increasing interest in the study of three-well systems (trimers) for a variety of reasons, such as

(i) its possible application in the construction of a BEC-transistor [20];

(ii) it is the simplest model which provides a bridge between the double-well and the multi-well systems $[46,47]$;

(iii) recent achievements in the experimental field, in particular the control promised by microtraps [48] suggest the realization of the trimer to be at hand [49].

\footnotetext{
3 The following identification has been done: $\beta_{1}=w ; \mu_{a}=w_{2} ; \mu_{c}=w_{1} ; \Omega=\alpha_{3} \beta_{2}$.

4 Notice, however, that it corresponds to a simplified three-well model, with no atom-atom interaction terms.
} 
It is easy to check that by combining the conserved quantity $I$ with the Hamiltonian $t(0)$ and choosing properly the coupling constants, we arrive at the following Hamiltonian ${ }^{5}$

$$
\mathcal{H}=\Omega_{2}\left(a_{2}^{\dagger} a_{1}+a_{1}^{\dagger} a_{2}+a_{2}^{\dagger} a_{3}+a_{3}^{\dagger} a_{2}\right)+\Omega\left(a_{1}^{\dagger} a_{3}+a_{3}^{\dagger} a_{1}\right)+\mu n_{1}+\mu n_{3}+\mu_{2} n_{2},
$$

which describes an array of three coupled wells, which will be referred to as the left (1), middle (2) and right (3) wells, respectively. Above, $\Omega$ (respectively $\Omega_{2}$ ) denote the tunneling of atoms between the left and the right wells (respectively the left-middle tunnelling and the middleright tunneling), while $\mu_{2}$ and $\mu$ are the external potentials. Obviously, adapting the choice of parameters, one can also treat the case where the left-middle and middle-right tunnellings are different.

For the particular case where $\Omega$ approaches to zero this model reduces to the asymmetric open trimer model in the absence of the interatomic scattering and a large external potential $\mu_{2}$ [49]. We observe here that if we consider this model in its full generality [49], with $\Omega_{2}$ and $\mu_{2}$ as adjustable parameters, a BEC-transistor [20] can be derived.

In the symmetric limit $\Omega=\Omega_{2}, \mu=\mu_{2}$ in (4.24) we recover the model of the three coupled BEC based on the $S U$ (3) symmetry, proposed by Milburn et al. [46] also in the absence of the interatomic scattering.

\subsubsection{Two-coupled BEC model with different types of atoms}

We consider the elementary monodromy matrices

$$
\begin{aligned}
& \Lambda^{[1]}(u)=\left(\begin{array}{ccc}
u+n_{a 1} & a_{1}^{\dagger} a_{2} & \alpha a_{1}^{\dagger} \\
a_{2}^{\dagger} a_{1} & u+n_{a 2} & \alpha a_{2}^{\dagger} \\
\alpha a_{1} & \alpha a_{2} & \alpha^{2}
\end{array}\right) \text { and } \Lambda^{[2]}(u)=\left(\begin{array}{ccc}
u+n_{b 1} & b_{1}^{\dagger} b_{2} & \beta b_{1}^{\dagger} \\
b_{2}^{\dagger} b_{1} & u+n_{b 2} & \beta b_{2}^{\dagger} \\
\beta b_{1} & \beta b_{2} & \beta^{2}
\end{array}\right), \\
& T(u)=\Lambda^{[1]}\left(u+w_{1}\right) \Lambda^{[2]}\left(u+w_{2}\right) .
\end{aligned}
$$

Then, the transfer matrix reads

$$
\begin{aligned}
t(u) \sim & u\left(n_{a}+n_{b}\right)+n_{a 1} n_{b 1}+n_{a 2} n_{b 2}+w_{1} n_{b}+w_{2} n_{a}+a_{1}^{\dagger} b_{2}^{\dagger} a_{2} b_{1}+a_{2}^{\dagger} b_{1}^{\dagger} a_{1} b_{2} \\
& +\Omega\left(a_{1}^{\dagger} b_{1}+a_{2}^{\dagger} b_{2}+b_{1}^{\dagger} a_{1}+b_{2}^{\dagger} a_{2}\right),
\end{aligned}
$$

where $\Omega=\alpha \beta$. It leads to the Hamiltonian $t(0)$ with conserved quantities $n_{j}=n_{a j}+n_{b j}, j=$ 1,2. This corresponds to a model of two wells ( $A$ and $B$ ) with $n_{j}$ atoms of type $j, j=1,2$. Here $n_{a j}\left(n_{b j}\right)$ denotes the number of atoms of type $j, j=1,2$ in the well $A(B)$. Basically, this Hamiltonian describes the tunneling of atoms of different types (1 and 2) in the two wells ( $A$ and $B$ ).

Notice that this Hamiltonian could also be interpreted as describing two wells ( $A$ and $B$ ) with two levels ( 1 and 2 ) in each well. Particles can tunnel between the wells and levels. The tunneling term $\Omega$ allow particles to tunnel between wells just in the same level. Similar models (fourmode Hamiltonians with tilted potentials) have been proposed recently in [50]. In this context we mention that multi-mode models are receiving more attention, specially in connection to the creation of a quantum computer from neutral atoms [50].

\footnotetext{
5 More specifically, $\mathcal{H}=t(0)+\alpha n$ and we have done the change of variables $\beta_{1} \rightarrow \beta ; \beta_{3} \rightarrow \beta ; \beta_{2} \rightarrow \gamma / \beta$, together with the following identification $\Omega_{2}=\gamma ; \Omega=\beta^{2} ; \mu=\left(\alpha-\beta^{2}\right) ; \mu_{2}=\left(\alpha-\gamma^{2} / \beta^{2}\right)$.
} 


\subsubsection{Creation/dissociation of a molecule with two conformations}

We consider the elementary monodromy matrices

$$
\begin{aligned}
& \Lambda^{[1]}(u)=\left(\begin{array}{ccc}
u+n_{A 1} & A_{1}^{\dagger} a_{1} & \alpha A_{1}^{\dagger} \\
a_{1}^{\dagger} A_{1} & u+n_{a 1} & \alpha a_{1}^{\dagger} \\
\alpha A_{1} & \alpha a_{1} & \alpha^{2}
\end{array}\right) \text { and } \\
& \Lambda^{[2]}(u)=\left(\begin{array}{ccc}
-\beta^{2} & \beta a_{2}^{\dagger} & \beta A_{2}^{\dagger} \\
\beta a_{2} & u-n_{a 2} & A_{2}^{\dagger} a_{2} \\
\beta A_{2} & a_{2}^{\dagger} A_{2} & u-n_{A 2}
\end{array}\right), \\
& T(u)=\Lambda^{[1]}\left(u+w_{1}\right) \Lambda^{[2]}\left(u+w_{2}\right),
\end{aligned}
$$

where we have used the sign-transposition and dilatation automorphisms on $\Lambda^{[2]}(u)$. Then, the transfer matrix reads

$$
\begin{aligned}
t(u) \sim & u\left(n_{a 1}-n_{a 2}\right)-n_{a 1} n_{a 2}-w_{1} n_{a 2}+w_{2} n_{a 1}-\alpha^{2} n_{A 2}-\beta^{2} n_{A 1} \\
& +\alpha \beta\left(A_{1}^{\dagger} A_{2}+A_{2}^{\dagger} A_{1}\right)+\alpha\left(a_{1}^{\dagger} a_{2}^{\dagger} A_{2}+A_{2}^{\dagger} a_{1} a_{2}\right)+\beta\left(a_{1}^{\dagger} a_{2}^{\dagger} A_{1}+A_{1}^{\dagger} a_{2} a_{1}\right)
\end{aligned}
$$

with conserved quantities $\delta n=n_{a 2}-n_{a 1}$ and $n_{\text {tot }}=2\left(n_{A 1}+n_{A 2}\right)+n_{a 1}+n_{a 2}$.

The Hamiltonian describes a molecule $A$, which exists in two conformations (two different stereochemical forms) $A_{1}$ and $A_{2}$, and is constituted with two atoms (or submolecules) $a_{1}$ and $a_{2}$. There are transitions between the two conformations of the molecule, and there is recombination/dissociation between the atoms and the two aspects of the molecule $A$. In this context, $\alpha$ and $\beta$ are related to the probabilities to obtain $A_{1}$ or $A_{2}$ starting from the atoms (or submolecules) $a_{1}$ and $a_{2}$. The relative proportion of atoms $\delta n$ and the total number of atoms in the system $n_{\text {tot }}$ are conserved.

\subsubsection{A generalized heteroatomic-molecular BEC model}

We consider the elementary monodromy matrices

$$
\begin{aligned}
& \Lambda^{[1]}(u)=\left(\begin{array}{ccc}
u+n_{a 1} & A^{\dagger} a_{2} & \alpha A^{\dagger} \\
a_{2}^{\dagger} A & u+n_{a 2} & \alpha a_{2}^{\dagger} \\
\alpha A & \alpha a_{2} & \alpha^{2}
\end{array}\right) \quad \text { and } \quad \Lambda^{[2]}(u)=\left(\begin{array}{ccc}
u+n_{a 1} & \beta a_{1}^{\dagger} & a_{1}^{\dagger} b_{2} \\
\beta a_{1} & \beta^{2} & \beta b_{2} \\
b_{2}^{\dagger} a_{1} & \beta b_{2}^{\dagger} & u+n_{b 2}
\end{array}\right), \\
& T(u)=\Lambda^{[1]}\left(u+w_{1}\right) \Lambda^{[2]}\left(u+w_{2}\right) .
\end{aligned}
$$

Then, the transfer matrix reads

$$
\begin{aligned}
t(u) \sim & u\left(n_{A}+n_{a 1}\right)+n_{A} n_{a 1}+w_{1} n_{a 1}+w_{2} n_{A}+\alpha^{2} n_{b 2}+\beta^{2} n_{a 2} \\
+ & \beta\left(A^{\dagger} a_{1} a_{2}+a_{2}^{\dagger} a_{1}^{\dagger} A\right)+\alpha \beta\left(a_{2}^{\dagger} b_{2}+b_{2}^{\dagger} a_{2}\right) .
\end{aligned}
$$

It leads to the Hamiltonian $t(0)$ with conserved quantity $n_{1}=n_{A}+n_{a 1}$. This model describes a molecule $A$ which can be dissociated into two different atoms $a_{1}$ and $a_{2}$. One of these atoms, $a_{2}$, can evolve to a different state $b_{2}$, which forbids the recombination to $A$. For instance, $A, a_{1}$ and $a_{2}$ can be trapped in one well, the transitions between $a_{2}$ and $b_{2}$ corresponding to a 'leak' of the $a_{2}$ atom toward a second well.

In the limit $\beta \rightarrow 0$ one recovers the model of Section 4.1.4. 


\subsubsection{Coupling of two oscillators with gl(3)}

For completeness we give here an example of coupling $g l(3)$ with oscillators. We take the case of two oscillators, but one can also couple a single oscillator, or three of them.

$$
\begin{aligned}
& \Lambda(u)=\left(\begin{array}{ccc}
u+n_{1} & a_{1}^{\dagger} a_{2} & \alpha a_{1}^{\dagger} \\
a_{2}^{\dagger} a_{1} & u+n_{2} & \alpha a_{2}^{\dagger} \\
\alpha a_{1} & \alpha a_{2} & \alpha^{2}
\end{array}\right) ; \quad L(u)=\left(\begin{array}{ccc}
u+e_{11} & e_{12} & e_{13} \\
e_{21} & u+e_{22} & e_{23} \\
e_{31} & e_{32} & u+e_{33}
\end{array}\right), \\
& T(u)=\Lambda(u) L(u) .
\end{aligned}
$$

The transfer matrix reads

$$
\begin{aligned}
t(u) \sim & u\left(n_{1}+n_{2}+e_{11}+e_{22}\right)+n_{1} e_{11}+n_{2} e_{22}+a_{1}^{\dagger} a_{2} e_{21}+a_{2}^{\dagger} a_{1} e_{12}+\alpha^{2} e_{33} \\
& +\alpha\left(a_{1}^{\dagger} e_{31}+a_{2}^{\dagger} e_{32}\right)+\alpha\left(a_{1} e_{13}+a_{2} e_{23}\right)+w_{1}\left(e_{11}+e_{22}\right)+w_{2}\left(n_{1}+n_{2}\right) .
\end{aligned}
$$

The conserved quantities are

$$
I_{j}=e_{j j}+n_{j}, \quad j=1,2 .
$$

\subsection{Coupling of one oscillator with $g l(N)$}

We take

$$
\begin{aligned}
& \Lambda(u)=\left(\begin{array}{cccc}
\mu u+n_{1} & \alpha_{2} a^{\dagger} & \ldots & \alpha_{N} a^{\dagger} \\
\alpha_{2} a & & & \\
\vdots & & \mathbb{M} & \\
\alpha_{N} a & & &
\end{array}\right) ; \quad L(u)=\left(\begin{array}{cccc}
u+e_{11} & e_{12} & \ldots & e_{1 N} \\
e_{21} & & & \\
\vdots & & u \mathbb{I}+\mathbb{E} & \\
e_{N 1} & & &
\end{array}\right) \\
& \text { with } \mathbb{M}_{i j}=\alpha_{i} \alpha_{j} \text { and } \mathbb{E}_{i j}=e_{i j}, \\
& T(u)=\Lambda(u) L(u) .
\end{aligned}
$$

Then, for $\mu=1$ and $\alpha$ 's real, one gets

$$
\begin{aligned}
t(u) \sim & u\left(e_{11}+n_{1}\right)+n_{1} e_{11}+\sum_{j=2}^{N}\left\{\alpha_{j}\left(a^{\dagger} e_{j 1}+e_{1, j} a\right)+\alpha_{j}^{2} e_{j j}\right\} \\
& +\sum_{2 \leqslant j<k \leqslant N} \alpha_{j} \alpha_{k}\left(e_{j k}+e_{k j}\right) .
\end{aligned}
$$

We have not used the parameters $w_{j}$, but from the form of $\Lambda(u)$ and $L(u)$, it is easy to see that one recovers them through the shifts $n_{1} \rightarrow n_{1}+w_{1}$ and $e_{j j} \rightarrow e_{j j}+w_{2}, j=1,2, \ldots, N$.

4.4. Two-coupled BEC model with $(N-1)$ levels

We consider $T(u)=\Lambda^{[1]}\left(u+w_{1}\right) \Lambda^{[2]}\left(u+w_{2}\right)$ with

$$
\begin{aligned}
& \Lambda_{j k}^{[1]}(u)=\delta_{j k} u+a_{j}^{\dagger} a_{k}, \quad j, k<N, \\
& \Lambda_{j N}^{[1]}(u)=\alpha_{N} a_{j}, \quad \Lambda_{N j}^{[2]}(u)=\alpha_{N} a_{j}^{\dagger}, \quad j<N \quad \text { and } \quad \Lambda_{N N}^{[1]}(u)=\alpha_{N}^{2}, \\
& \Lambda_{j k}^{[2]}(u)=\delta_{j k} u+b_{j}^{\dagger} b_{k}, \quad j, k<N,
\end{aligned}
$$




$$
\Lambda_{j N}^{[2]}(u)=\beta_{N} b_{j}, \quad \Lambda_{N j}^{[2]}(u)=\beta_{N} b_{j}^{\dagger}, \quad j<N \quad \text { and } \quad \Lambda_{N N}^{[2]}(u)=\beta_{N}^{2},
$$

where $b_{j}, b_{j}^{\dagger}$ is another set of oscillators. The transfer matrix $t(u)=\operatorname{tr} T(u)$ now reads

$$
\begin{aligned}
t(u) \sim & \sum_{j=1}^{N-1}\left\{u\left(n_{j}^{[2]}+n_{j}^{[1]}\right)+n_{j}^{[2]} n_{j}^{[1]}+\alpha_{N} \beta_{N}\left(a_{j}^{\dagger} b_{j}+b_{j}^{\dagger} a_{j}\right)\right\} \\
& +\sum_{1 \leqslant j \neq k \leqslant N-1} a_{j}^{\dagger} a_{k} b_{k}^{\dagger} b_{j},
\end{aligned}
$$

where $n_{j}^{[1]}=a_{j}^{\dagger} a_{j}$ and $n_{j}^{[2]}=b_{j}^{\dagger} b_{j}$. It leads to the 'fundamental' Hamiltonians

$$
\begin{aligned}
t(0) & \sim \sum_{j=1}^{N-1}\left(n_{j}^{[2]}+n_{j}^{[1]}\right), \\
t^{\prime}(0) & \sim \sum_{j=1}^{N-1}\left\{n_{j}^{[2]} n_{j}^{[1]}+\alpha_{N} \beta_{N}\left(a_{j}^{\dagger} b_{j}+b_{j}^{\dagger} a_{j}\right)\right\}+\sum_{1 \leqslant j \neq k \leqslant N-1} a_{j}^{\dagger} a_{k} b_{k}^{\dagger} b_{j} .
\end{aligned}
$$

Again, the shifts $n_{j}^{[p]} \rightarrow n_{j}^{[p]}+w_{p}, j=1,2, \ldots, N-1, p=1,2$ give back the $w$ dependence.

It corresponds to a generalization of the previous Hamiltonian (4.26), which could be interpreted, for example, as describing two wells with $N-1$ levels in each well. The quantities $I_{j}=n_{j}^{[1]}+n_{j}^{[2]}, j=1,2, \ldots, N-1$, are conserved.

\section{5. $N$-coupled BEC model}

We consider the elementary monodromy matrices

$$
\begin{aligned}
& \Lambda_{j k}^{[1]}(u)=\delta_{j k} u+a_{j} a_{k}^{\dagger}, \quad j, k=1,2, \\
& \Lambda_{j k}^{[1]}(u)=\alpha_{k} a_{j}, \quad \Lambda_{k j}^{[1]}(u)=\alpha_{k} a_{j}^{\dagger}, \quad j=1,2, k=3, \ldots, N, \\
& \Lambda_{j k}^{[1]}(u)=\alpha_{k} \alpha_{j}, \quad j, k=3, \ldots, N, \\
& \Lambda_{j k}^{[2]}(u)=\alpha_{k} \alpha_{j}, \quad j, k=1,2, \\
& \Lambda_{j k}^{[2]}(u)=\delta_{j k} u+a_{j}^{\dagger} a_{k}, \quad j, k=3, \ldots, N, \\
& \Lambda_{j k}^{[2]}(u)=\alpha_{j} a_{k}, \quad \Lambda_{k j}^{[2]}(u)=\alpha_{j} a_{k}^{\dagger}, \quad j=1,2, k=3, \ldots, N .
\end{aligned}
$$

The transfer matrix takes the form

$$
t(u) \sim \frac{1}{2} \sum_{i=1}^{2} \sum_{\substack{j=1 \\ j \neq i}}^{N} \alpha_{i} \alpha_{j}\left(a_{i}^{\dagger} a_{j}+a_{j}^{\dagger} a_{i}\right)+\sum_{i=1}^{N} \alpha_{i}^{2} n_{i} .
$$

It corresponds to a multi-well system, which generalizes the previous Hamiltonian (4.23). The total number operator $\sum_{i=1}^{N} n_{i}$ is conserved. 


\section{Bethe ansatz equations}

We use the results of [51], applied to the special representations we are studying. This can be done when the representations are lowest weight. However, when the representations are reducible, the Bethe ansatz will give the eigenvalues only on the irreducible parts. It has to be applied for each lowest weights.

For the spin chain part, associated to the elementary monodromy matrix $L(u)$, one has always a unique lowest weight $v$ defining the representation (the spin) one is working with. When dealing with oscillators, the Fock vacuum $|0\rangle$ is a natural lowest weight, but different cases can appear, as we shall see.

\section{1. $N=2$ case}

For $N=2$, the elementary monodromy matrices (4.1) and (4.2) become all triangular when applied to the Fock vacuum $|0\rangle$ and/or to the lowest weight vector $v_{s}$ :

$$
\begin{aligned}
& L(u) v_{s}=\left(\begin{array}{cc}
u+s & S_{+} \\
0 & u-s
\end{array}\right) v_{s}, \quad s \in \frac{1}{2} \mathbb{Z}_{+}, \quad \mathcal{L}(u)|0\rangle=\left(\begin{array}{cc}
u & 0 \\
0 & u
\end{array}\right)|0\rangle, \\
& \Lambda(u)|0\rangle=\left(\begin{array}{cc}
u & \beta a^{\dagger} \\
0 & \beta^{2}
\end{array}\right)|0\rangle, \quad \hat{\Lambda}(u)|0\rangle=\left(\begin{array}{cc}
\beta^{2} & \beta a^{\dagger} \\
0 & u
\end{array}\right)|0\rangle .
\end{aligned}
$$

Note that $\mathcal{L}(u)$ is proportional to the identity matrix, because the representation is reducible. Indeed all the vectors $|p\rangle=\left(a_{2}^{\dagger}\right)^{p}|0\rangle, p \in \mathbb{Z}_{+}$, are lowest weight vectors for $\mathcal{L}(u)$ :

$$
\mathcal{L}(u)|p\rangle=\left(\begin{array}{cc}
u+p & a_{1}^{\dagger} a_{2} \\
0 & u
\end{array}\right)|p\rangle .
$$

Then, depending on the model we are studying, we will get as pseudo-vacuum for the monodromy matrix, either the total Fock vacuum $|0,0\rangle=|0\rangle \otimes|0\rangle$, or different combinations of the type $|p, q\rangle=|p\rangle \otimes|q\rangle,\left|v_{s}, p\right\rangle=v_{s} \otimes|p\rangle$, etc. When several pseudo-vacua are at our disposal, we will have to repeat the Bethe ansatz method (described below) for each of the pseudo-vacuum in order to get a complete set of eigenvalues for the transfer matrix.

In all cases, the total monodromy matrix is triangular, and the pseudo-vacuum obeys:

$$
\begin{aligned}
& T_{j j}^{L L}(u)\left|v_{s}, v_{r}\right\rangle=\lambda_{j}(u)\left|v_{s}, v_{r}\right\rangle, \quad j=1,2 \quad \text { with }\left\{\begin{array}{l}
\lambda_{1}(u)=\left(u+w_{1}+s\right)\left(u+w_{2}+r\right), \\
\lambda_{2}(u)=\left(u+w_{1}-s\right)\left(u+w_{2}-r\right),
\end{array}\right. \\
& T_{j j}^{L \mathcal{L}}(u)\left|v_{s}, p\right\rangle=\lambda_{j}(u)\left|v_{s}, p\right\rangle, \quad\left\{\begin{array}{l}
j=1,2 \\
p \in \mathbb{Z}_{+}
\end{array}\right. \\
& \text {with }\left\{\begin{array}{l}
\lambda_{1}(u)=\left(u+w_{1}+s\right)\left(u+w_{2}+p\right), \\
\lambda_{2}(u)=\left(u+w_{1}-s\right)\left(u+w_{2}\right)
\end{array}\right. \\
& T_{j j}^{\mathcal{L} \mathcal{L}}(u)|p, q\rangle=\lambda_{j}(u)|p, q\rangle, \quad\left\{\begin{array}{l}
j=1,2 \\
p, q \in \mathbb{Z}_{+}
\end{array}\right. \\
& \text {with }\left\{\begin{array}{l}
\lambda_{1}(u)=\left(u+w_{1}+p\right)\left(u+w_{2}+q\right) \\
\lambda_{2}(u)=\left(u+w_{1}\right)\left(u+w_{2}\right)
\end{array}\right. \\
& T_{j j}^{L \Lambda}(u)\left|v_{s}, 0\right\rangle=\lambda_{j}(u)\left|v_{s}, 0\right\rangle, \quad j=1,2 \quad \text { with }\left\{\begin{array}{l}
\lambda_{1}(u)=\left(u+w_{1}+s\right)\left(u+w_{2}\right), \\
\lambda_{2}(u)=\alpha^{2}\left(u+w_{1}-s\right),
\end{array}\right.
\end{aligned}
$$




$$
\begin{aligned}
& T_{j j}^{\mathcal{L} \Lambda}(u)|p, 0\rangle=\lambda_{j}(u)|p, 0\rangle, \quad\left\{\begin{array} { l } 
{ j = 1 , 2 , } \\
{ p \in \mathbb { Z } _ { + } }
\end{array} \quad \text { with } \left\{\begin{array}{l}
\lambda_{1}(u)=\left(u+w_{1}+p\right)\left(u+w_{2}\right), \\
\lambda_{2}(u)=\alpha^{2}\left(u+w_{1}\right)
\end{array}\right.\right. \\
& T_{j j}^{\Lambda \Lambda}(u)|0,0\rangle=\lambda_{j}(u)|0,0\rangle, \quad j=1,2 \quad \text { with }\left\{\begin{array}{l}
\lambda_{1}(u)=\left(u+w_{1}\right)\left(u+w_{2}\right), \\
\lambda_{2}(u)=\alpha^{2} \beta^{2}
\end{array}\right.
\end{aligned}
$$

It implies that the pseudo-vacuum is an eigenvector of the transfer matrix. We will summarize these different cases in the notation

$$
t(u)|\Omega\rangle=\left(\lambda_{1}(u)+\lambda_{2}(u)\right)|\Omega\rangle,
$$

where $|\Omega\rangle$ is one of the pseudo-vacuum(s).

The other eigenvalues of the transfer matrix then read

$$
\lambda(u)=A_{0}(u) \lambda_{1}(u)+A_{1}(u) \lambda_{2}(u),
$$

where the dressing functions $A_{j}(u)$ are given by:

$$
A_{0}(u)=\prod_{n=1}^{M} \frac{u-u_{n}+\frac{1}{2}}{u-u_{n}-\frac{1}{2}} \quad \text { and } \quad A_{1}(u)=\prod_{n=1}^{M} \frac{u-u_{n}-\frac{3}{2}}{u-u_{n}-\frac{1}{2}} .
$$

The parameters $u_{n}, 1 \leqslant n \leqslant M$, are the Bethe roots, their number $M$ being also a free parameter. All these parameters are determined by the Bethe equations

$$
\prod_{\substack{m=1 \\ m \neq n}}^{M} \frac{u_{n}-u_{m}-1}{u_{n}-u_{m}+1}=\frac{\lambda_{1}\left(u_{n}+\frac{1}{2}\right)}{\lambda_{2}\left(u_{n}+\frac{1}{2}\right)}, \quad 1 \leqslant n \leqslant M .
$$

\section{2. $N=3$ case}

The existence of a pseudo-vacuum is not ensured anymore. For instance in the example treated in Section 4.2.2, one easily computes the action of $t(u)$ on the Fock vacuum:

$$
t(u)|0,0\rangle=\left(u^{2}+\beta_{1}^{2} u+\beta_{1}^{2} \alpha_{3}^{2}\right)|0,0\rangle+\beta_{1}^{*} \beta_{2}^{*} \alpha_{3} b^{\dagger}|0,0\rangle
$$

so that the vacuum is an eigenvector of the transfer matrix only if the condition $\beta_{1} \beta_{2} \alpha_{3}=0$ is satisfied. Note that this condition is weaker than demanding $|0,0\rangle$ to be a lowest weight vector for the monodromy matrix, which would lead to $\alpha_{3}=0$.

\subsubsection{Pseudo-vacua}

We give the eigenvalues $\lambda_{j}(u)$ of the pseudo-vacuum (when it exists) under the generators $T_{j j}(u), j=1,2,3$. We order them according to the section where they are presented. The pseudovacua are built on the Fock vacuum $|0\rangle \equiv|0\rangle \otimes \cdots \otimes|0\rangle$ of the models.

Model 5.2.1. One must impose the condition $\beta_{1} \beta_{2}=0$. In that case, there are several pseudovacua $|p\rangle=\left(b^{\dagger}\right)^{p}|0,0,0\rangle, p \in \mathbb{Z}_{+}$,

$$
T_{11}(u)|p\rangle=u^{2}|p\rangle ; \quad T_{22}(u)|p\rangle=\beta_{1}^{2}(u+p)|p\rangle ; \quad T_{33}(u)|p\rangle=\alpha_{3}^{2} \beta_{2}^{2}|p\rangle .
$$

Model 5.2.2. One must impose the condition $\beta_{1} \beta_{2}=0$. In that case, the Fock vacuum has eigenvalues

$$
T_{11}(u)|0\rangle=\beta_{1}^{2} u^{2}|0\rangle ; \quad T_{22}(u)|0\rangle=\beta_{2}^{2} u^{2}|0\rangle ; \quad T_{33}(u)|0\rangle=\beta_{3}^{2} u^{2}|0\rangle .
$$


Model 5.2.3. The Fock vacuum has eigenvalues

$$
T_{11}(u)|0\rangle=u^{2}|0\rangle ; \quad T_{22}(u)|0\rangle=u^{2}|0\rangle ; \quad T_{33}(u)|0\rangle=\alpha^{2} \beta^{2}|0\rangle .
$$

Model 5.2.4. There are two types of pseudo-vacua

$$
|p\rangle=\left(a_{2}^{\dagger}\right)^{p}|0,0,0,0\rangle \quad \text { and } \quad|-p\rangle=\left(b_{1}^{\dagger}\right)^{p}|0,0,0,0\rangle, \quad p \in \mathbb{Z}_{+} .
$$

Gathering them in the notation $|p\rangle, p \in \mathbb{Z}$, their eigenvalues read

$$
\begin{aligned}
& T_{11}(u)|p\rangle=\beta^{2} u|p\rangle ; \quad T_{22}(u)|p\rangle=u(u+p)|p\rangle ; \\
& T_{33}(u)|p\rangle=\alpha^{2} u|p\rangle, \quad p \in \mathbb{Z} .
\end{aligned}
$$

Model 5.2.5. One must impose the condition $\alpha \beta=0$. In that case, the Fock vacuum has eigenvalues

$$
T_{11}(u)|0\rangle=u^{2}|0\rangle ; \quad T_{22}(u)|0\rangle=\beta^{2} u|0\rangle ; \quad T_{33}(u)|0\rangle=\alpha^{2} u|0\rangle .
$$

Model 5.2.6. We denote by $v$ the $g l(3)$ lowest weight vector, with eigenvalues $\left(\lambda_{1}, \lambda_{2}, \lambda_{3}\right)$ under the $g l(3)$ Cartan generators. The pseudo-vacuum is $|v\rangle=|0\rangle \otimes v$, with eigenvalues

$$
\begin{aligned}
& T_{11}(u)|v\rangle=u\left(u+\lambda_{1}\right)|v\rangle ; \quad T_{22}(u)|v\rangle=u\left(u+\lambda_{2}\right)|v\rangle ; \\
& T_{33}(u)|v\rangle=\alpha^{2}\left(u+\lambda_{3}\right)|v\rangle .
\end{aligned}
$$

\subsubsection{Bethe equations}

Now that we have determined the conditions for the existence of a pseudo-vacuum, one can write

$$
t(u)|\Omega\rangle=\left(\lambda_{1}(u)+\lambda_{2}(u)+\lambda_{3}(u)\right)|\Omega\rangle,
$$

where the eigenvalues $\lambda_{j}(u)$ can be read in Section 5.2.1. Then, the other transfer matrix eigenvalues read

$$
\lambda(u)=A_{0}(u) \lambda_{1}(u)+A_{1}(u) \lambda_{2}(u)+A_{2}(u) \lambda_{3}(u)
$$

with the dressing functions

$$
\begin{aligned}
& A_{0}(u)=\prod_{n=1}^{M^{(1)}} \frac{u-u_{n}^{(1)}+\frac{1}{2}}{u-u_{n}^{(1)}-\frac{1}{2}}, \\
& A_{1}(u)=\prod_{n=1}^{M^{(1)}} \frac{u-u_{n}^{(1)}-\frac{3}{2}}{u-u_{n}^{(1)}-\frac{1}{2}} \prod_{n=1}^{M^{(2)}} \frac{u-u_{n}^{(2)}}{u-u_{n}^{(2)}-1}, \\
& A_{2}(u)=\prod_{n=1}^{M^{(2)}} \frac{u-u_{n}^{(2)}-2}{u-u_{n}^{(2)}-1} .
\end{aligned}
$$

We have here two sets of Bethe roots, $u_{n}^{(1)}, 1 \leqslant n \leqslant M^{(1)}$, and $u_{n}^{(2)}, 1 \leqslant n \leqslant M^{(2)}$, constrained by the Bethe equations

$$
\prod_{\substack{m=1 \\ m \neq n}}^{M^{(1)}} \frac{u_{n}^{(1)}-u_{m}^{(1)}-1}{u_{n}^{(1)}-u_{m}^{(1)}+1} \prod_{m=1}^{M^{(2)}} \frac{u_{n}^{(1)}-u_{m}^{(2)}+\frac{1}{2}}{u_{n}^{(1)}-u_{m}^{(2)}-\frac{1}{2}}=\frac{\lambda_{1}\left(u_{n}^{(1)}+\frac{1}{2}\right)}{\lambda_{2}\left(u_{n}^{(1)}+\frac{1}{2}\right)}, \quad 1 \leqslant n \leqslant M^{(1)},
$$




$$
\prod_{m=1}^{M^{(1)}} \frac{u_{n}^{(2)}-u_{m}^{(1)}+\frac{1}{2}}{u_{n}^{(2)}-u_{m}^{(1)}-\frac{1}{2}} \prod_{\substack{m=1 \\ m \neq n}}^{M^{(2)}} \frac{u_{n}^{(2)}-u_{m}^{(2)}-1}{u_{n}^{(2)}-u_{m}^{(2)}+1}=\frac{\lambda_{2}\left(u_{n}^{(2)}+1\right)}{\lambda_{3}\left(u_{n}^{(2)}+1\right)}, \quad 1 \leqslant n \leqslant M^{(2)}
$$

\subsection{General case}

For the general case of $N \times N$ matrices, and supposing the existence of pseudo-vacua $|\Omega\rangle$ (possibly with conditions on the parameters of the models, as above), we will get for the eigenvalues of the transfer matrix

$$
\lambda(u)=\sum_{k=1}^{N} A_{k-1}(u) \lambda_{k}(u)
$$

with pseudo-vacuum eigenvalues

$$
T_{k k}(u)|\Omega\rangle=\lambda_{k}(u)|\Omega\rangle, \quad k=1, \ldots, N \quad \text { so that } t(u)|\Omega\rangle=\left(\sum_{k=1}^{N} \lambda_{k}(u)\right)|\Omega\rangle
$$

and dressing functions

$$
A_{k}(u)=\prod_{n=1}^{M^{(k)}} \frac{u-u_{n}^{(k)}-\frac{k+2}{2}}{u-u_{n}^{(k)}-\frac{k}{2}} \prod_{n=1}^{M^{(k+1)}} \frac{u-u_{n}^{(k+1)}-\frac{k-1}{2}}{u-u_{n}^{(k+1)}-\frac{k+1}{2}}, \quad 0 \leqslant k \leqslant N-1,
$$

with $M^{(0)}=M^{(N)}=0$.

The $N-1$ types of Bethe roots $u_{n}^{(k)}, 1 \leqslant n \leqslant M^{(k)}, 1 \leqslant k \leqslant N-1$, will be determined by the Bethe equations:

$$
\begin{aligned}
& \prod_{m=1}^{M^{(k-1)}} \frac{u_{n}^{(k)}-u_{m}^{(k-1)}+\frac{1}{2}}{u_{n}^{(k)}-u_{m}^{(k-1)}-\frac{1}{2}} \prod_{\substack{m=1 \\
m \neq n}}^{M^{(k)}} \frac{u_{n}^{(k)}-u_{m}^{(k)}-1}{u_{n}^{(k)}-u_{m}^{(k)}+1} \prod_{m=1}^{M^{(k+1)}} \frac{u_{n}^{(k)}-u_{m}^{(k+1)}+\frac{1}{2}}{u_{n}^{(k)}-u_{m}^{(k+1)}-\frac{1}{2}} \\
& =\frac{\lambda_{k}\left(u_{n}^{(k)}+\frac{k}{2}\right)}{\lambda_{k+1}\left(u_{n}^{(k)}+\frac{k}{2}\right)}, \quad 1 \leqslant n \leqslant M^{(k)} \text { and } 1 \leqslant k \leqslant N-1 .
\end{aligned}
$$

They depend on the model and pseudo-vacuum through the eigenvalues $\lambda_{k}(u), k=1, \ldots, N$.

\section{Superalgebras and fermions}

We have seen that the Yangian is not sufficient to build BEC models based on fermions. For such a purpose, one needs to consider another algebraic structure, the super-Yangian $Y(M \mid N)$ based on the superalgebra $g l(M \mid N)$.

The models look very similar to the ones presented in Section 4, with the notable difference that the choices for the fermionic oscillators is not the same, allowing more flexibility in the construction.

\subsection{The superalgebra $\operatorname{gl}(M \mid N)$}

To define a superalgebra, one needs to introduce a grading $[\cdot]$ that will distinguish the fermionic generators from the bosonic ones. For $g l(M \mid N)$, denoting $e_{i j}, i, j=1, \ldots, M+N$, these 
generators, the grading is defined as ${ }^{6}$

$$
\left[e_{i j}\right]=[i]+[j] \quad \text { with }[k]= \begin{cases}0 & \text { if } 1 \leqslant k \leqslant M \\ 1 & \text { if } M+1 \leqslant k \leqslant M+N .\end{cases}
$$

The generators with grading 0 are bosonic ones; they form a $g l(M) \oplus g l(N)$ subalgebra of $g l(M \mid N)$, generated by $e_{i j}$, with $i, j \leqslant M$ and $i, j>M$. The remaining generators are of fermionic type, and form a representation $(N, \bar{M}) \oplus(\bar{N}, M)$ of this subalgebra. The supercommutator reads

$$
\left[e_{i j}, e_{k l}\right\}=-(-1)^{([i]+[j])([k]+[l])}\left[e_{k l}, e_{i j}\right\}=\delta_{j k} e_{i l}-(-1)^{([i]+[j])([k]+[l])} \delta_{i l} e_{k j}
$$

which amounts to consider commutators when $e_{i j}$ and/or $e_{k l}$ are/is bosonic (i.e. of grade 0 ), and anti-commutators when both are fermionic.

The fundamental representation is of dimension $M+N$ :

$$
\pi\left(e_{i j}\right)=E_{i j},
$$

where now $E_{i j}$ are graded elementary matrices of size $M+N$.

\subsection{The super-Yangian $Y(M \mid N)$}

One defines the super-Yangian through a graded $R$-matrix, obeying a graded version of YBE. By graded version of YBE, we mean that one has to use a graded tensor product on the auxiliary spaces:

$$
\left(E_{i j} \otimes E_{k l}\right) \cdot\left(E_{p q} \otimes E_{r s}\right)=(-1)^{([k]+[l])([p]+[q])}\left(E_{i j} E_{p q}\right) \otimes\left(E_{k l} E_{r s}\right),
$$

where the grading is the same as the one of $\operatorname{gl}(M \mid N)$.

In fact, everything looks formally the same as for the Yangian, with the restriction that one has to take care of the grading (6.1). For instance, the $R$-matrix reads:

$$
R_{12}(x)=\mathbb{I} \otimes \mathbb{I}-\frac{1}{x} P_{12},
$$

with now the super-permutation operator

$$
P_{12}=\sum_{i, j=1}^{M+N}(-1)^{[j]} E_{i j} \otimes E_{j i}=\sum_{i=1}^{M+N}\left(\sum_{j=1}^{M} E_{i j} \otimes E_{j i}-\sum_{j=M+1}^{M+N} E_{i j} \otimes E_{j i}\right) .
$$

Plugging this $R$-matrix in the relation (2.1), and taking care of the graded tensor product leads to

$$
\left[L_{i j}(u), L_{k l}(v)\right\}=\frac{(-1)^{[i]([k]+[l])+[k][l]}}{u-v}\left(L_{k j}(u) L_{i l}(v)-L_{k j}(v) L_{i l}(u)\right) .
$$

We will use elementary monodromy matrices built on $g l(M \mid N)$ :

$$
L_{i j}(u)=u \delta_{i j}+e_{i j}, \quad e_{i j} \in g l(M \mid N) .
$$

\footnotetext{
6 Other choices of grading could be used, but we will stick to this one throughout the paper.
} 


\subsection{Transfer matrix and symmetries}

The monodromy matrix $\Delta^{(L)} T(u)=T^{[1]}(u) \cdots T^{[L]}(u)$ gives a transfer matrix of the form

$$
\operatorname{st}(u)=\operatorname{str}\left(T^{[1]}(u) \cdots T^{[L]}(u)\right),
$$

where the super-trace of a matrix is defined by

$$
\operatorname{str}(A)=\sum_{i=1}^{M+N}(-1)^{[i]} A_{i i}=\sum_{i=1}^{M} A_{i i}-\sum_{i=M+1}^{M+N} A_{i i} \quad \text { for } A=\sum_{i, j=1}^{M+N} A_{i j} E_{i j} .
$$

As for the bosonic case, one can show that

$$
[s t(u), s t(v)]=0 .
$$

Again, to get Hermitian Hamiltonian, we will focus on the case $L=2$, so that the monodromy and transfer matrices we will be concerned of, have the form

$$
\begin{aligned}
& T_{k l}(v)=\sum_{n=0}^{2} v^{n} T_{k l}^{(n)} \quad \text { with } T_{k l}^{(2)}=\omega_{k} \delta_{k l} \text { and } \omega_{k} \in \mathbb{C}, \\
& s t(u)=t_{2} u^{2}+t_{1} u+t_{0} \quad \text { with } t_{2} \in \mathbb{C} .
\end{aligned}
$$

Specializing to $g l(M \mid N)$ representations will give different models. These models will have also a symmetry, as one can see from the relation

$$
\left[s t(u), T_{k l}^{(1)}\right]=\left(\omega_{k}-\omega_{l}\right)\left(T_{k l}^{(0)}+u T_{k l}^{(1)}\right)
$$

proving again that the quantities

$$
I_{k}=T_{k k}^{(1)} \quad \forall k=1, \ldots, N,
$$

commute with the transfer matrix.

We will be essentially interested in the oscillator representation

$$
\pi\left(e_{i j}\right)=a_{i}^{\dagger} a_{j} \quad \text { with }\left[a_{i}, a_{j}^{\dagger}\right\}=\mu_{i} \delta_{i j},
$$

where $\left(a_{i}, a_{i}^{\dagger}\right)$ for $1 \leqslant i \leqslant M$ (respectively $\left.M+1 \leqslant i \leqslant M+N\right)$ are bosons (respectively fermions), i.e. $\left[a_{i}\right]=\left[a_{i}^{\dagger}\right]=[i]$.

This choice of grading implies that $\left[e_{i j}\right]=\left[a_{i}^{\dagger}\right]+\left[a_{j}\right]=[i]+[j]$, in accordance with the gradation of $g l(M \mid N)$. It corresponds to a bosonic (respectively fermionic) oscillator representation for the $g l(M)$ (respectively $g l(N)$ ) subalgebra. Remark that the opposite choice (i.e. $\left.\left[a_{i}\right]=\left[a_{i}^{\dagger}\right]=[i]+1\right)$ is also possible.

The inhomogeneous oscillator monodromy matrices will then be obtained by taking constant bosonic oscillators. In that process, the choice of the grading for the oscillators will be essential, since it will determine which of the oscillators can be possibly set to constant.

Despite of the grading, the elementary matrices are still Hermitian. Hence, the models will be Hermitian. 


\section{Fermionic BEC models}

\subsection{Two by two (graded) matrices}

We are dealing with the $g l(1 \mid 1)$ case, and we focus on the elementary monodromy matrices of the form

$$
\begin{array}{ll}
\mathcal{L}(u)=\left(\begin{array}{c|c}
u+n_{1} & a_{1}^{\dagger} a_{2} \\
\hline a_{2}^{\dagger} a_{1} & u-n_{2}
\end{array}\right), \\
\Lambda(u)=\left(\begin{array}{c|c}
u+n & \beta c^{\dagger} \\
\hline \beta c & \beta^{2}
\end{array}\right), \quad \hat{\Lambda}(u)=\left(\begin{array}{c|c}
-\beta^{2} & \beta c \\
\hline \beta c^{\dagger} & u-n
\end{array}\right),
\end{array}
$$

where in $\mathcal{L}(u)$ one of the couple $\left(a_{j}, a_{j}^{\dagger}\right), j=1,2$, is bosonic and the other one fermionic, while in $\Lambda(u)$ and $\hat{\Lambda}(u),\left(c, c^{\dagger}\right)$ is fermionic.

\subsubsection{Fermionic heteroatomic BEC model}

We consider $\operatorname{str} \mathcal{L}^{[1]}\left(u+w_{1}\right) \mathcal{L}^{[2]}\left(u+w_{2}\right)$, with oscillators $\left(a_{1}, c_{1}\right)$ for $\mathcal{L}^{[1]}(u)$ and $\left(c_{2}, a_{2}\right)$ for $\mathcal{L}^{[2]}(u)$. One can consider either $\left(a_{1}, a_{2}\right)$ to be bosonic and $\left(c_{1}, c_{2}\right)$ fermionic, or $\left(a_{1}, a_{2}\right)$ fermionic and $\left(c_{1}, c_{2}\right)$ bosonic.

The transfer matrix reads:

$$
\begin{aligned}
s t(u) \sim & u\left(n_{a 1}+n_{a 2}+n_{c 1}+n_{c 2}\right)+n_{a 1} n_{c 1}-n_{a 2} n_{c 2}+w_{1}\left(n_{c 1}+n_{c 2}\right) \\
& +w_{2}\left(n_{a 1}+n_{a 2}\right)+a_{1}^{\dagger} c_{2}^{\dagger} c_{1} a_{2}+a_{2}^{\dagger} c_{1}^{\dagger} c_{2} a_{1} .
\end{aligned}
$$

We get a fermionic version of the model described in Section 4.1.3. It can be interpreted as modelizing a coupled pair of one boson and one fermion which can tunnel together from one well to another.

\subsubsection{Heteroatomic-molecular BEC model with fermions}

We consider $\operatorname{str} \mathcal{L}\left(u+w_{1}\right) \Lambda\left(u+w_{2}\right)$, with oscillators $\left(a_{1} \equiv b, a_{2} \equiv a\right)$ for $\mathcal{L}(u)$ and $c$ for $\Lambda(u)$. In $\mathcal{L}(u)$, we take $b$ bosonic. Then, $a$ is fermionic, as well as is $c$ in $\Lambda(u)$. We get

$$
s t(u) \sim u\left(n_{b}+n_{c}\right)+n_{b} n_{c}+w_{1} n_{c}+w_{2} n_{b}+\beta^{2} n_{a}+\beta\left(a^{\dagger} c^{\dagger} b-b^{\dagger} a c\right) .
$$

We find a new version of the heteroatomic-molecular BEC model of Section 4.1.4, with now a bosonic molecule $b$ constituted of two fermionic atoms $a$ and $c$.

The shift automorphism applied on $b$ produces additional terms in the transfer matrix

$$
H_{\text {bound }} \sim\left(\beta\left(b+b^{\dagger}\right)+\beta^{2}\right) n_{c}+w_{2} \beta\left(b+b^{\dagger}\right)+\alpha \beta\left(a^{\dagger} c^{\dagger}-a c\right) .
$$

\subsubsection{Fermionic two-wells}

We start with $\Lambda^{[1]}\left(u+w_{1}\right)$ and $\Lambda^{[2]}\left(u+w_{2}\right)$, set all the bosons to constant, keeping the fermions $c_{1}$ and $c_{2}$.

$$
\begin{aligned}
\operatorname{str} \Lambda^{[1]}\left(u+w_{1}\right) \Lambda^{[2]}\left(u+w_{2}\right)= & \left(u+w_{1}+n_{c 1}\right)\left(u+w_{2}+n_{c 2}\right) \\
& -\beta_{1} \beta_{2} c_{1}^{\dagger} c_{2}+\beta_{1} \beta_{2} c_{1} c_{2}^{\dagger}, \\
\operatorname{st}(u) \sim u\left(n_{c 1}+n_{c 2}\right)+n_{c 1} n_{c 2}+ & w_{1} n_{c 2}+w_{2} n_{c 1}-\beta_{1} \beta_{2}\left(c_{1}^{\dagger} c_{2}+c_{2}^{\dagger} c_{1}\right) .
\end{aligned}
$$


One recognizes a two-wells models, but now with atoms of fermionic nature.

We mention here that there has been recently a great interest in Bose-Einstein condensates with fermions since the achievement of quantum degeneracy in ultracold Fermi gases (see, for example [18] and references therein).

\subsection{Three by three (graded) matrices}

We are now dealing with the $g l(2 \mid 1)$ case, corresponding to $3 \times 3$ matrices. Again the number of possible models becomes numerous, so that we present the generic case and treat only two examples, physically relevant.

\subsubsection{Generic case}

We present here a general formulation for the transfer matrix, which encompasses all the possible models, by setting some of the (bosonic) oscillators to constant.

The two generic elementary monodromy matrices have the form

$$
\begin{aligned}
& L^{[1]}(u)=\left(\begin{array}{cc|c}
u_{1}+n_{1} & a_{1}^{\dagger} a_{2} & a_{1}^{\dagger} a_{3} \\
a_{2}^{\dagger} a_{1} & u_{2}+n_{2} & a_{2}^{\dagger} a_{3} \\
\hline a_{3}^{\dagger} a_{1} & a_{3}^{\dagger} a_{2} & u_{3}+n_{3}
\end{array}\right) \quad \text { and } \\
& L^{[2]}(u)=\left(\begin{array}{cc|c}
v_{1}+m_{1} & c_{1}^{\dagger} c_{2} & c_{1}^{\dagger} c_{3} \\
c_{2}^{\dagger} c_{1} & v_{2}+m_{2} & c_{2}^{\dagger} c_{3} \\
\hline c_{3}^{\dagger} c_{1} & c_{3}^{\dagger} c_{2} & v_{3}+m_{3}
\end{array}\right)
\end{aligned}
$$

with the notations

$$
n_{j}=a_{j}^{\dagger} a_{j} \quad \text { and } \quad m_{j}=c_{j}^{\dagger} c_{j}, \quad j=1,2,3 .
$$

For $L^{[1]}(u)$ to be of $g l(2 \mid 1)$ type, one can choose either $a_{1}$ and $a_{2}$ bosonic and $a_{3}$ fermionic, or $a_{1}$ and $a_{2}$ fermionic and $a_{3}$ bosonic. Obviously, the same criteria apply for $L^{[2]}(u), c_{j}, j=1,2,3$.

When the oscillators are bosonic, one can choose to set them to constant: in that case the corresponding spectral parameter $\left(u_{j}\right.$ or $\left.v_{j}\right)$ has to be set to zero. In all other cases, it is set to $u$. For instance, if $a_{1}$, bosonic, is the only one set to a constant $\alpha_{1}$, then one has $u_{1}=0$ and $u_{2}=u_{3}=v_{1}=v_{2}=v_{3}=u$. We will also have in this case $a_{1}^{\dagger}=\alpha_{1}^{*}$ and $n_{1}=\left|\alpha_{1}\right|^{2}$.

Keeping these rules in mind, one can compute a generic transfer matrix. It takes the form (up to irrelevant constant terms)

$$
\begin{aligned}
& s t(u)=\sum_{j=1}^{3}\left(u_{j} m_{j}+v_{j} n_{j}\right)+H, \\
& H=a_{1}^{\dagger} a_{2} c_{2}^{\dagger} c_{1}+c_{1}^{\dagger} c_{2} a_{2}^{\dagger} a_{1}+a_{1}^{\dagger} a_{3} c_{3}^{\dagger} c_{1}+c_{1}^{\dagger} c_{3} a_{3}^{\dagger} a_{1}+a_{2}^{\dagger} a_{3} c_{3}^{\dagger} c_{2}+c_{2}^{\dagger} c_{3} a_{3}^{\dagger} a_{2}+\sum_{j=1}^{3} m_{j} n_{j} .
\end{aligned}
$$

The 'true' Hamiltonian $s t(0)=H$ of a given model is then obtained through the above rules, after choosing which of the oscillators are bosonic or fermionic, and, among the bosonic ones, which of them are set to constant. 
The conserved quantities of the model take the form

$$
u I_{j}=u_{j} m_{j}+v_{j} n_{j}, \quad j=1,2,3 .
$$

Of course, depending of the choices, some of these quantities can be zero after use of the rules.

Let us also remark that if one takes all the oscillators to be bosonic or fermionic (a situation forbidden in the case of $g l(2 \mid 1)$ ), one gets a generic transfer matrix for the usual three by three matrices, as treated in Section 4.2.

\subsubsection{A fermionic two-coupled BEC}

To exemplify the above techniques, we construct a fermionic version of the model given in Section 4.2.3.

We take $a_{3}$ and $c_{3}$ to be bosonic, and set both of them to constant, $\alpha$ and $\gamma$ (both real) respectively. Then, one gets four couples of oscillators, all of them being fermionic. The rules lead to

$$
\begin{array}{lll}
u_{1}=u_{2}=v_{1}=v_{2}=u & \text { and } \quad u_{3}=v_{3}=0, \\
a_{3}=a_{3}^{\dagger}=\alpha \in \mathbb{R} \quad \text { and } & n_{3}=\alpha^{2}, \\
c_{3}=c_{3}^{\dagger}=\gamma \in \mathbb{R} \quad \text { and } & m_{3}=\gamma^{2} .
\end{array}
$$

Thus, we get an Hamiltonian

$$
H=a_{1}^{\dagger} a_{2} c_{2}^{\dagger} c_{1}+c_{1}^{\dagger} c_{2} a_{2}^{\dagger} a_{1}+\alpha \gamma\left(a_{1}^{\dagger} c_{1}+c_{1}^{\dagger} a_{1}+a_{2}^{\dagger} c_{2}+c_{2}^{\dagger} a_{2}\right)+m_{1} n_{1}+m_{2} n_{2}
$$

with conserved quantities

$$
I_{1}=n_{1}+m_{1} \quad \text { and } \quad I_{2}=n_{2}+m_{2} .
$$

We recover the Hamiltonian of Section 4.2.3, with the notable difference that the atoms have a fermionic nature.

\subsubsection{Fermionic three coupled BEC model}

If now one takes $a_{3}$ bosonic and constant $(\alpha \in \mathbb{R})$, and $c_{1}$ and $c_{2}$ bosonic and constant $\left(\gamma_{j} \in \mathbb{R}\right)$, one gets:

$$
\begin{aligned}
& u_{1}=u_{2}=v_{3}=u \quad \text { and } \quad u_{3}=v_{1}=v_{2}=0, \\
& a_{3}=a_{3}^{\dagger}=\alpha \in \mathbb{R} \quad \text { and } \quad n_{3}=\alpha^{2}, \\
& c_{j}=c_{j}^{\dagger}=\gamma_{j} \in \mathbb{R} \quad \text { and } \quad m_{j}=\gamma_{j}^{2}, \quad j=1,2,
\end{aligned}
$$

leading to (using the sign-transposition and dilatation automorphisms on $L^{[2]}(u)$ )

$$
\begin{aligned}
H= & \gamma_{1} \gamma_{2}\left(a_{1}^{\dagger} a_{2}+a_{2}^{\dagger} a_{1}\right)+\alpha \gamma_{1}\left(a_{1}^{\dagger} c_{3}+c_{3}^{\dagger} a_{1}\right)+\alpha \gamma_{2}\left(a_{2}^{\dagger} c_{3}+c_{3}^{\dagger} a_{2}\right) \\
& +\gamma_{1}^{2} n_{1}+\gamma_{2}^{2} n_{2}-\alpha m_{3} .
\end{aligned}
$$

We get the fermionic version of the Hamiltonian described in Section 4.2.2. 


\section{Conclusion}

In the BEC context, we have constructed integrable generalized models in a systematic way exploring different representations of the $g l(N)$ algebra and the $g l(M \mid N)$ superalgebra. Some existing models, such as the two-site Bose-Hubbard model, have been recovered and new ones have been predicted. Interestingly, a two-coupled BEC model with a field, a three-coupled BEC model and a two-coupled BEC-model with different types of atoms, among others, have been introduced. The use of the $g l(M \mid N)$ superalgebra allows the introduction of fermions, leading to systems mixing bosons and fermions, as they are presently studied in condensed matter BEC experiments. In this context, the 'integrable approach' can be viewed as a technique to construct in a very general way Hamiltonians relevant for these studies.

The energy spectrum of these models has been derived, through the Bethe ansatz equations, by the use of analytical Bethe ansatz. The next step in the study of these systems in this general framework, is the determination of the (Bethe) eigenstates and eigenfunctions, which would allow to investigate the classical and quantum dynamics of such systems.

Finally, we remark that more general integrable models can be obtained using the method presented in the present work. They are constructed using products of more elementary monodromy matrices, with the restriction that the Hermiticity of their Hamiltonian is not guaranteed anymore. Apart from the trial and error method that one can use on a case-by-case basis, a general analysis determining the conditions under which Hamiltonians are Hermitian would certainly improve the landscape of integrable BEC models.

\section{Acknowledgements}

A.F. thanks R.R.B. Correia, J. Links and A.P. Tonel for useful discussions. A.F. also acknowledges support from PRONEX under contract CNPq 66.2002/1998-99 and CNPq (Conselho Nacional de Desenvolvimento Científico e Tecnológico). E.R. wishes to thank the Instituto de Física da Universidade Federal do Rio Grande do Sul (Porto Alegre) for hospitality during this work was completed.

\section{Appendix A. Quantum determinant and conserved quantities}

We have seen that the expansion of the monodromy matrix provides some conserved quantities of the integrable models. However, other conserved quantities can be obtained when considering the center of the algebra. For the Yangian, it is known that its center is generated by the quantum determinant [52]:

$$
q \operatorname{det}(u)=\sum_{\sigma \in S_{N}} \operatorname{sgn}(\sigma) T_{1, \sigma(1)}(u) T_{2, \sigma(2)}(u-1) \cdots T_{N, \sigma(N)}(u-N+1),
$$

where $S_{N}$ is the group of permutations. It is clear that the conserved quantities obtained in this way are quite complicated, but they may be of some help for the study of the different models. To illustrate this, we give the form of the quantum determinant when $N=2$ and 3

$$
\begin{aligned}
q \operatorname{det}(u)= & T_{11}(u) T_{22}(u-1)-T_{12}(u) T_{21}(u-1) \quad \text { for } N=2, \\
q \operatorname{det}(u)= & T_{11}(u) T_{22}(u-1) T_{33}(u-2)+T_{12}(u) T_{23}(u-1) T_{31}(u-2) \\
& +T_{13}(u) T_{21}(u-1) T_{32}(u-2)-T_{12}(u) T_{21}(u-1) T_{33}(u-2)
\end{aligned}
$$




$$
-T_{13}(u) T_{22}(u-1) T_{32}(u-2)-T_{11}(u) T_{23}(u-1) T_{32}(u-2) \quad \text { for } N=3 \text {, }
$$

$q \operatorname{det}(u)$ is a polynomial in $u$, of degree $2 N$ since $T(u)$ is of degree two. Hence, one gets a priori $2 N$ conserved quantities. They are not all independent, but they can provide new conserved quantities, not contained in the transfer matrix, nor given by (3.6). Remark that these quantities are a priori not Hermitian, but, since they are central in the whole Yangian, so are their adjoint. Hence, one can build Hermitian (and anti-Hermitian) conserved quantities from $q \operatorname{det}(u)$.

Indeed, for $N=2$, expanding $q \operatorname{det}(u)$ from the expansion (3.2), one gets (up to constant terms)

$$
\begin{aligned}
& q \operatorname{det}(u)=\sum_{n=0}^{3} d_{n} u^{n}, \\
& d_{3}=\omega_{1} T_{22}^{(1)}+\omega_{2} T_{11}^{(1)}, \\
& d_{2}=\omega_{1} T_{22}^{(1)}-2 \omega_{2} T_{11}^{(1)}+\omega_{1} T_{22}^{(0)}+\omega_{2} T_{11}^{(0)}-T_{12}^{(1)} T_{21}^{(1)}, \\
& d_{1}=\omega_{2} T_{11}^{(1)}-2 \omega_{2} T_{11}^{(0)}+T_{11}^{(1)} T_{22}^{(0)}+T_{11}^{(0)} T_{22}^{(1)}-T_{12}^{(1)} T_{21}^{(0)}-T_{12}^{(0)} T_{21}^{(1)}, \\
& d_{0}=\omega_{2} T_{11}^{(1)}-T_{11}^{(0)} T_{22}^{(1)}+T_{12}^{(0)} T_{21}^{(1)}
\end{aligned}
$$

with $\omega_{k}=\mu_{a k} \mu_{b k}, k=1,2$, when dealing with oscillator representations, or $\omega_{k}=1$ for $g l(N)$ representations.

After some algebras, and using the conserved quantities (3.6), one obtains the following invariants

$$
\begin{aligned}
& c_{0}=T_{12}^{(0)} T_{21}^{(1)}+T_{11}^{(0)}\left(\omega_{2}-T_{22}^{(1)}\right), \\
& c_{1}=T_{12}^{(1)} T_{21}^{(0)}+T_{22}^{(0)}\left(\omega_{1}-T_{11}^{(1)}\right), \\
& c_{2}=T_{12}^{(1)} T_{21}^{(1)}-\omega_{1} T_{22}^{(0)}-\omega_{2} T_{11}^{(0)} .
\end{aligned}
$$

Of course, the explicit form of these invariant will depend on the representations we will use, i.e. on the physical model we are studying.

In the case $N=3$, the same kind of calculation leads to more complicated expressions. The simplest ones read:

$$
\begin{aligned}
c_{4}= & \omega_{3} T_{12}^{(1)} T_{21}^{(1)}+\omega_{2} T_{13}^{(1)} T_{31}^{(1)}+\omega_{1} T_{23}^{(1)} T_{32}^{(1)}-\omega_{2} \omega_{3} T_{11}^{(0)} \\
& -\omega_{1} \omega_{3} T_{22}^{(0)}-\omega_{1} \omega_{2} T_{33}^{(0)}, \\
c_{0}= & T_{11}^{(0)} T_{22}^{(0)} T_{33}^{(0)}-T_{11}^{(0)} T_{23}^{(0)} T_{32}^{(0)}-T_{12}^{(0)} T_{21}^{(0)} T_{33}^{(0)}+T_{12}^{(0)} T_{23}^{(0)} T_{31}^{(0)} \\
& +T_{13}^{(0)} T_{21}^{(0)} T_{32}^{(0)}-T_{13}^{(0)} T_{22}^{(0)} T_{31}^{(0)} .
\end{aligned}
$$

\section{References}

[1] M.T. Batchelor, X.-W. Guan, N. Oelkers, A. Foerster, Thermal and magnetic properties of integrable spin-1 and spin-3/2 chains with applications to real compounds, J. Stat. Mech. 0410 (2004) P017.

[2] M.T. Batchelor, X.-W. Guan, N. Oelkers, K. Sakai, Z. Tsuboi, A. Foerster, Exact results for thermal and magnetic properties of strong coupling ladder compounds, Phys. Rev. Lett. 91 (2003) 217202;

M.T. Batchelor, X.-W. Guan, N. Oelkers, Z. Tsuboi, Integrable models and quantum spin ladders: Comparison between theory and experiment for the strong coupling compounds, cond-mat/0512489. 
[3] C.T. Black, D.C. Ralph, M. Tinkham, Spectroscopy of the superconducting gap in individual nanometer-scale aluminum particles, Phys. Rev. Lett. 76 (1996) 688;

C.T. Black, D.C. Ralph, M. Tinkham, Gauge-voltage studies of discrete electronic states in aluminum nanoparticles, Phys. Rev. Lett. 78 (1997) 4087;

J. von Delft, D.C. Ralph, Spectroscopy of discrete energy levels in ultrasmall metallic grains, Phys. Rep. 345 (2001) 61.

[4] R.J. Baxter, Exactly Solved Models in Statistical Mechanics, Academic Press, 1982.

[5] F.H. Essler, V.E. Korepin, Exactly Solvable Models of Strongly Correlated Electrons, World Scientific, 1994.

[6] L.D. Faddeev, The Bethe Ansatz, Andrejeroski Lectures, 1993, SFB288 preprint 70.

[7] F. Iachello, A. Arimo, The Interacting Boson Model, Cambridge Univ. Press, 1995.

[8] A.J. Leggett, Bose-Einstein condensation in the alkali gases: Some fundamental concepts, Rev. Mod. Phys. 73 (2001) 307.

[9] L. Lipatov, High energy asymptotics of multi-colour QCD and exactly solvable lattice models, JETP Lett. 59 (1994) 596, hep-th/9311037.

[10] L. Faddeev, G. Korchemsky, High energy QCD as a completely integrable model, Phys. Lett. B 342 (1995) 311, hep-th/9404173.

[11] G. Ferretti, R. Heise, K. Zarembo, New integrable structures in large- $N$ QCD, hep-th/0404187.

[12] A.V. Belitsky, V.M. Braun, A.S. Gorsky, G.P. Korchemsky, Integrability in QCD and beyond, Int. J. Mod. Phys. A 19 (2004) 4715, hep-th/0407232.

[13] J.A. Minahan, K. Zarembo, The Bethe-Ansatz for $N=4$ super-Yang-Mills, JHEP 0303 (2003) 013, hep-th/ 0212208 .

[14] N. Beisert, M. Staudacher, The $N=4$ SYM integrable super spin chain, Nucl. Phys. B 670 (2003) 439;

N. Beisert, M. Staudacher, Long-Range PSU(2,214) Bethe Ansaetze for Gauge Theory and Strings, Nucl. Phys. B 727 (2005) 1, hep-th/0504190.

[15] S.N. Bose, Plancks Gesetz und Lichtquantenhypothese, Z. Phys. 26 (1924) 178;

A. Einstein, Quantentheorie des einatomigen idealen Gases, Sitzungsber. Preussichen Akad. Wiss. Phys.-Math. K1. (1924) 261.

[16] E.A. Cornell, C.E. Wieman, Nobel lecture: Bose-Einstein condensation in a dilute gas, the first 70 years and some recent experiments, Rev. Mod. Phys. 74 (2002) 875;

J.R. Anglin, W. Ketterle, Bose-Einstein condensation of atomic gases, Nature 416 (2002) 211.

[17] P. Zoller, Bose-Einstein condensation: Making it with molecules, Nature 417 (2002) 493.

[18] J. Hutson, P. Soldán, Molecule formation in ultracold atomic gases, physics/0607234.

[19] M. Héritier, In search of exact solutions, Nature 414 (2001) 31;

M.T. Batchelor, The Bethe ansatz after 75 years, Phys. Today 60 (2007) 36.

[20] J. Stickney, A. Zozulya, D. Anderson, Transistor-like behaviour of a Bose-Einstein condensate in a triple well potential, cond-mat/0607706.

[21] E.K. Sklyanin, L.D. Faddeev, Quantum mechanical approach to completely integrable models of field theory, Dokl. Acad. Nauk SSSR 243 (1978) 1430;

E.K. Sklyanin, L.D. Faddeev, Method of the inverse scattering problem and quantum nonlinear Schrödinger equation, Dokl. Acad. Nauk SSSR 244 (1978) 1337.

[22] E.K. Sklyanin, Quantum version of the method of inverse scattering problem, Zap. Nauchn. Sem. LOMI 95 (1980) 55.

[23] P.P. Kulish, N.Yu. Reshetikhin, E.K. Sklyanin, Yang-Baxter equation and representation theory: I, Lett. Math. Phys. 5 (1981) 393.

[24] P.P. Kulish, E.K. Sklyanin, Quantum inverse scattering method and the Heisenberg ferromagnet, Phys. Lett. A 70 (1979) 461.

[25] L.D. Faddeev, N.Yu. Reshetikhin, L.A. Takhtajan, Quantization of Lie groups and Lie algebras, Leningrad Math. J. 1 (1990) 193.

[26] V.E. Korepin, New effects in the massive Thirring model: Repulsive case, Commun. Math. Phys. 76 (1980) 165; V.E. Korepin, G. Izergin, N.M. Bogoliubov, Quantum Inverse Scattering Method, Correlation Functions and Algebraic Bethe Ansatz, Cambridge Univ. Press, 1993.

[27] L.D. Faddeev, L.A. Takhtajan, Spectrum and scattering of excitations in the one-dimensional isotropic Heisenberg model, J. Sov. Math. 24 (1984) 241;

L.D. Faddeev, L.A. Takhtajan, What is the spin of a spin wave? Phys. Lett. A 85 (1981) 375.

[28] P. Kulish, N.Yu. Reshetikhin, Diagonalisation of $G L(N)$ invariant transfer matrices and quantum N-wave system (Lee model), J. Phys. A 16 (1983) L591. 
[29] E. Ogievetsky, P. Wiegmann, Factorized S-matrix and the Bethe ansatz for simple Lie groups, Phys. Lett. B 168 (1986) 360.

[30] V.G. Drinfel'd, Hopf algebras and the quantum Yang-Baxter equation, Sov. Math. Dokl. 32 (1985) 254;

V.G. Drinfel'd, A new realization of Yangians and quantized affine algebras, Sov. Math. Dokl. 36 (1988) 212.

[31] R.J. Baxter, Partition function of the eight-vertex lattice model, Ann. Phys. 70 (1972) 193;

R.J. Baxter, J. Stat. Phys. 8 (1973) 25.

[32] C.N. Yang, Some exact results for the many-body problem in one dimension with repulsive delta-function interaction, Rev. Lett. 19 (1967) 1312.

[33] M.-L. Ge, K. Xue, Y. Wang, An Introduction to Yangian in Physics, Second Pacific Winter School in Theoretical Physics, Korea, 1995, cond-mat/9509090.

[34] A. Kundu, Yang-Baxter algebra and generation of quantum integrable models, nlin.SI/0609001.

[35] G. Ortiz, R. Somma, J. Dukelsky, S. Rombouts, Exactly-solvable models derived from a generalized Gaudin algebra, Nucl. Phys. B 707 (2005) 421.

[36] E.T. Jaynes, F.W. Cummings, Proc. IEEE 51 (1963) 89.

[37] D. Jonathan, K. Furuya, A. Vidiella-Barranco, Dressed-state approach to population trapping in the JaynesCummings model, quant-ph/9904067, and references therein.

[38] H. Haken, H. Wolf, W.D. Brewer, The Physics of Atoms and Quanta: Introduction to Experiments and Theory, Springer, 2004.

[39] J. Links, H.-Q. Zhou, R.H. McKenzie, M.D. Gould, Algebraic Bethe ansatz method for the exact calculation of energy spectra and form factors: Applications to models of Bose-Einstein condensates in metallic nanograins, J. Phys. A 36 (2003) R63;

A. Foerster, J. Links, H.-Q. Zhou, Exact Solvability in Contemporary Physics, Classical and Quantum Nonlinear Integrable Systems, IOP Publ., 2003.

[40] H.-Q. Zhou, J. Links, M.D. Gould, R.H. McKenzie, Solvable models of Bose-Einstein condensates: A new algebraic Bethe ansatz scheme, J. Math. Phys. 44 (2003) 4690, cond-mat/0211249.

[41] M. Duncan, A. Foerster, J. Links, E. Mattei, N. Oelkers, A. Tonel, Emergent quantum phases in a heteronuclear molecular Bose-Einstein condensate model, Nucl. Phys. B 767 (2007) 227, quant-ph/0610244;

L. Zhou, W. Zhang, H.Y. Ling, H. Pu, Quantum correlation in the photoassociation of a heteronuclear Bose-Einstein condensate, quant-ph/0611005.

[42] H.-Q. Zhou, J. Links, R.H. McKenzie, X.-W. Guan, Exact results for a tunnel-coupled pair of trapped Bose-Einstein condensates, J. Phys. A 36 (2003) L113, cond-mat/0203009.

[43] G.J. Milburn, J. Corney, E.M. Wright, D.F. Walls, Quantum dynamics of an atomic Bose-Einstein condensate in a double-well potential, Phys. Rev. A 55 (1997) 4318.

[44] A.P. Tonel, J. Links, A. Foerster, Quantum dynamics of a model for two Josephson-coupled Bose-Einstein condensates, J. Phys. A 38 (2005) 1235.

[45] M. Albiez, R. Gati, J. Fölling, S. Hunsmann, M. Cristiani, M.K. Oberthaler, Direct observation of tunneling and nonlinear self-trapping in a single bosonic Josephson junction, Phys. Rev. Lett. 95 (2005) 010402.

[46] K. Nemoto, C. Holmes, G. Milburn, W. Munro, Quantum dynamics of three coupled atomic Bose-Einstein condensates, Phys. Rev. A 63 (2001) 013604-1.

[47] B. Liu, L.-B. Fu, S.-P. Yang, J. Liu, Josephson oscillation and transition to self-trapping for Bose-Einsteincondensates in a triple-well trap, cond-mat/0610200.

[48] H. Ott, et al., Bose-Einstein condensation in a surface microtrap, Phys. Rev. Lett. 87 (2001) 230401; W. Hänsel, et al., Bose-Einstein condensation on a microelectronic chip, Nature 413 (2001) 498;

J. Reichel, Microchip traps and Bose-Einstein condensation, Appl. Phys. B 75 (2002) 469.

[49] P. Buonsante, R. Franzosi, V. Pena, Dynamical instability in a trimeric chain of interacting Bose-Einstein condensates, Phys. Rev. Lett. 90 (2003) 050404.

[50] D.R. Dounas-Frazer, A.M. Hermundstad, L.D. Carr, Ultracold bosons and entanglement in the tilted double-well, quant-ph/0609119.

[51] D. Arnaudon, N. Crampé, A. Doikou, L. Frappat, E. Ragoucy, Analytical Bethe Ansatz for closed and open $g l(n)-$ spin chains in any representation, J. Stat. Mech. 02 (2005) P007, math-ph/0411021.

[52] A. Molev, M. Nazarov, G. Olshanski, Yangians and classical Lie algebras, Russ. Math. Survey 51 (1996) 205 , hep-th/9409025. 